\title{
QUELS LIEUX POUR LA MÉDIATION CIVILE EN EUROPE?1
}

Evelyne Serverin

Diretora de Pesquisa do Centre National de la Recherche Scientifique, Paris, França. Doutora em Direito. eserveri@u-paris10.fr

RESUMO: O presente estudo visa a examinar a mediação civil na Europa, abordando os principais desafios à aplicação do instituto na atualidade.

PALAVRAS-CHAVE: Mediação; Europa; Direito Processual; Poder Jurisdicional.

RÉSUMÉ: Cette étude a l'objectif d'examiner la médiation civile en Europe en abordant ces principaux défis aujourd'hui.

MOTS-CLÉS: Médiation; Europe; Droit Processuel; Pouvoir Jurisditionnel.

\section{1 - Définitions}

Parmi les activités dont la finalité est de procurer aux citoyens une issue à leurs litiges celles qui impliquent l'intervention amiable d'un tiers reçoivent aujourd'hui une faveur toute particulière dans les sociétés occidentales. Et cette faveur est accrue s'agissant de litiges civils, dans lesquels les parties sont à même de disposer librement de leurs droits. Cette intervention amiable se distingue des formes autoritaires de règlement des litiges qui sont accomplies par une autorité publique (un juge), ou une autorité privée (l'arbitre). Ce tiers «qui ne juge pas» peut être invité à intervenir dans un litige à des titres divers: comme magistrat, agissant dans sa mission de conciliation en dehors de ses pouvoirs juridictionnels, comme personne qualifiée désignée par le juge (dans le cadre du procès), comme commission ou instance aménagée par le droit étatique en vue de contribuer au règlement de litiges particuliers, comme personne privée contractuellement investie d'une mission de rapprochement par des parties à un

\footnotetext{
${ }^{1}$ Artigo recebido em 10/02/2015 e aprovado em 08/05/2015.
} 
Revista Eletrônica de Direito Processual - REDP. Volume 15. Janeiro a Junho de 2015 Periódico Semestral da Pós-Graduação Stricto Sensu em Direito Processual da UERJ. Patrono: José Carlos Barbosa Moreira. www.redp.com.br ISSN 1982-7636 PP 107-149

litige. Quel que soit le nom sous lequel on le désigne (médiateur ou conciliateur) ce tiers intervenant à titre amiable a pour seule mission de tenter d'aider les parties à trouver un terrain d'entente, et ne pourra, au mieux, que constater les termes d'un accord s'il en est établi un.

Dans le cadre de ce rapport, le terme de «médiation » sera utilisé pour désigner toutes les formes d'activité de traitement non juridictionnel des conflits (y compris la conciliation), et à quelque moment que ces activités se situent (avant ou après l'introduction d'une instance). Ainsi définie, la médiation se distingue du jugement, de l'arbitrage, et de l'amiable composition, toutes activités qui impliquent un règlement autoritaire du différend. Cette définition «fonctionnelle» permet d'éviter l'écueil des différences terminologiques qui se rencontrent entre les droits internes des Etats.

2- Réalisations du Conseil de l'Europe en matière de règlement amiable

Les motifs de la préférence pour les formes amiables d'intervention des tiers dans les litiges sont divers: économie des fonds publics consacrés à la justice, conviction d'une meilleure efficacité de ces procédés en termes de rapprochement des parties, adhésion aux thèses d'un pluralisme juridique qui oppose les règlements négociés et les règlements imposés. Quels que soient les motifs retenus, on constate que les Etats multiplient les signes d'intérêt pour ces types d'action.

Cet intérêt se manifeste au plan communautaire, notamment avec le réseau extrajudiciaire européen, et les travaux en cours de la Commission européenne sur un livre vert en matière de modes alternatifs de règlement des litiges. Au sein du Conseil de l'Europe ces efforts permettent d'envisager sur ce point une coordination des instruments juridiques des 41 états membres.

Les activités de règlement amiable ont fait l'objet de nombreuses actions promotionnelles par le Conseil de l'Europe au cours des vingt dernières années, au travers des activités de ses différentes composantes, Comité des Ministres, Comité Européen de Coopération juridique (CDCJ), Comités d'experts et comités conventionnels. Une partie de ces actions a donné lieu d'ores et déjà à des recommandations; d'autres présentent une dimension exploratoire à laquelle se rattache le présent rapport. 


\section{2-1- Les recommandations}

Les diverses recommandations du Conseil de l'Europe prises au cours des vingt dernières années en matière de justice ont comporté des observations relatives à l'intervention amiable dans les litiges.

1- Au titre de l'accès à la justice, la Recommandation $\mathrm{n}^{\circ} \mathrm{R}(81) 7 \mathrm{du}$ Comité des ministres a fait figurer parmi les procédés de simplification de cet accès « les mesures facilitant ou encourageant dans les cas appropriés la conciliation des parties et le règlement amiable des différends, avant toute procédure judiciaire ou au cours d'une procédure engagée $»$.

2- Dans la perspective d'un allègement de la charge des tribunaux, la recommandation $n^{\circ} \mathrm{R}(86) 12$ du Comité des Ministres a placé en tête des mesures préconisées «le règlement amiable des différends, soit en dehors de l'ordre judiciaire, soit avant ou pendant la procédure judiciaire ».

3- Pour les personnes en situation de grande pauvreté, il été recommandé d'assurer «l'accès effectif aux modes para-judiciaires de solution des conflits (...), telles que la médiation et la conciliation (...), en étendant le bénéfice de l'aide judiciaire ou toute autre forme d'assistance à ces modes de solution des conflits » (Recommandation $\left.\mathrm{n}^{\circ} \mathrm{R}(93) 1\right)$.

4- Les pouvoirs des juges en matière de règlement amiable n'ont pas été oubliés, puisque la recommandation $\mathrm{n}^{\circ} \mathrm{R}$ (94)12 sur l'indépendance, l'efficacité et le rôle des juges a fait figurer dans la liste des «responsabilités judiciaires », (principe V-3 e) «l'encouragement des parties à obtenir un règlement amiable ». Ce même « encouragement au règlement amiable » a été adressé par la recommandation $n^{\circ} \mathrm{R}(95)$ 5 , article $6 \mathrm{~g}$, au juge intervenant sur recours en matière civile et commerciale.

5- Au cours des années les plus récentes, les procédures amiables ont fait l'objet de valorisation dans des secteurs du droit particuliers : Recommandation $\mathrm{n}^{\circ} \mathrm{R}(98) 1$ sur la médiation familiale, suivie d'une Conférence européenne sur le droit de la famille consacrée à la "Médiation familiale en Europe", (Strasbourg, 1-2oct. 1998); Recommandation $\mathrm{N}^{\circ}(99) 19$ sur la médiation en matière pénale. De même, le Groupe de projet sur le droit administratif (CJ-DA) a achevé l'examen d'un projet de 
Revista Eletrônica de Direito Processual - REDP. Volume 15. Janeiro a Junho de 2015 Periódico Semestral da Pós-Graduação Stricto Sensu em Direito Processual da UERJ. Patrono: José Carlos Barbosa Moreira. www.redp.com.br ISSN 1982-7636 PP 107-149

Recommandation sur les modes alternatifs de règlement des litiges entre les autorités administratives et les personnes privées ${ }^{2}$.

$\mathrm{Au}$ fil des recommandations, la médiation et autres procédures amiables ont quitté les coulisses de la scène judiciaire pour devenir une figure emblématique de la justice moderne et efficace. Curieuse destinée pour une forme de règlement des différends qui était traditionnellement considérée comme un signe de défiance envers la justice professionnelle!

Quoiqu'il en soit de cet étrange retour de l'histoire, il semble aujourd'hui que se fasse sentir le besoin de procéder à une réflexion d'ensemble sur les procédures amiables, après l'adoption dans l'enthousiasme de recommandations en leur faveur.

2-2- Le temps de la réflexion

L'activité d'expertise s'intensifie autour des règlements dits «alternatifs des litiges », et particulièrement de ceux qui impliquent l'intervention amiable de tiers dans les litiges.

- Le séminaire multilatéral sur les Modes Alternatifs de Règlement des Litiges, qui s'est déroulé à Strasbourg du 29 novembre au $1^{\text {er }}$ décembre 1999 dans le cadre des activités pour le développement et la consolidation de la stabilité démocratique (ADACS), a été l'occasion d'aborder une série de questions relatives à ces procédés, notamment les définitions, la qualité des tiers intervenants, la nécessité d'une réglementation, l'incidence des cultures juridiques, et l'efficacité des actions ${ }^{3}$. Mais les rapports remis par les différents intervenants sont difficiles à synthétiser, notamment en raison de l'absence de grille de questionnement commun et l'absence de systématisation dans l'approche. Selon les cas, le raisonnement a été mené par secteur (famille, consommation) ou par pays (France, Royaume-Uni, Norvège, Pays -Nordiques) dans une perspective d'information plus que d'évaluation.

2 Le texte proposé vise à promouvoir l'utilisation par les Etats membres de ces modes dans les litiges administratifs, comme alternative viable aux procédures contentieuses, et qui peuvent contribuer à la réduction de la surcharge des tribunaux et au rapprochement de l'administration avec le public. L'annexe de ce projet de Recommandation prévoit un certain nombre de principes de bonnes pratiques pour chacun des modes examinés (révision interne, conciliation, médiation, règlement amiable, arbitrage) que les Etats devraient suivre dans leurs droits et leurs pratiques nationaux.

3 Jagtenberg R. W, [2000], Rapport Général au Séminaire Multilatéral sur les Modes Alternatifs de Règlement des Litiges, Strasbourg. 
Revista Eletrônica de Direito Processual - REDP. Volume 15. Janeiro a Junho de 2015 Periódico Semestral da Pós-Graduação Stricto Sensu em Direito Processual da UERJ. Patrono: José Carlos Barbosa Moreira. www.redp.com.br ISSN 1982-7636 PP 107-149

- Le comité d'experts mandaté par le CDJC pour mettre au point un guide législatif des mesures visant à accroître l'efficacité de la justice (CJ-EJ ) a mis à son programme un chapitre sur "la résolution alternative des litiges » (Alternative Dispute Resolution $)^{4}$. Avec le projet de mandat révisé de ce comité, ce sont désormais trois groupes qui travailleront en parallèle, l'un sur le fonctionnement du système judiciaire, le second sur les procédures alternatives de règlement des litiges, le troisième sur la consultation et l'assistance juridique ${ }^{5}$.

Ainsi ces procédés tendent à occuper une place centrale dans le dispositif d'amélioration des systèmes judiciaires des différents pays. Mais en donnant aux ADR une place de choix parmi les procédés visant à accroître l'efficacité de la justice, le comité se trouvait confronté à la nécessité de justifier également de l'efficacité de ces procédés. En effet, si les ADR ont pour vocation d'éviter le recours au tribunal, on ne sait ni dans quelles conditions, ni à quel coût, ni avec quel effet, ils fonctionnent. Contrairement à l'activité des tribunaux, dont la connaissance est assurée dans tous les pays par un système statistique plus ou moins élaboré 6 , les actions de règlement hors jugement ne sont observées, ni confrontées à d'autres pratiques. Le besoin de connaissance dans le domaine des procédés alternatifs s'accroît en même temps que $s$ 'intensifie la promotion qui leur est faite.

Cependant, la conception d'enquêtes empiriques sur ce terrain se heurte à de nombreuses difficultés, dont la moindre n'est pas le caractère dispersé des secteurs concernés et l'absence de données statistiques. Dans ce contexte, il est important de souligner la nécessité d'évaluer, sur la base de critères statistiques et de moyens d'évaluation communs, l'impact de la médiation sur la résolution des litiges en matière civile. Ce besoin de connaissance a été signalé par la Résolution $\mathrm{N}^{\circ} 1$ «Rendre la justice au $21^{\circ}$ siècle », adoptée par les Ministres européens de la justice lors de leur 23e Conférence à Londres les 8-9 juin 2000. A la lumière de cette Résolution, les travaux futurs du CJ-EJ pourraient utilement examiner la question des avantages comparatifs de la médiation pour résoudre des litiges en matière civile dans les différents pays.

4 Conseil de l'Europe, Groupe de travail du Comité d'experts sur l'efficience de la justice, Strasbourg, 30 juin-2 juillet 1999. Compte rendu du 12 juillet 1999.

5 CJ-EJ, projet de mandat révisé, 26 juin 2000.

6 Pour une présentation du système pour la France, v. Serverin E. [1993], "Lire les statistiques judiciaires hier et aujourd'hui", Bulletin du Centre Pierre Léon d'histoire Economique et sociale, 1, p. 4353. 
Revista Eletrônica de Direito Processual - REDP. Volume 15. Janeiro a Junho de 2015

Periódico Semestral da Pós-Graduação Stricto Sensu em Direito Processual da UERJ.

Patrono: José Carlos Barbosa Moreira. www.redp.com.br ISSN 1982-7636 PP 107-149

D'ores et déjà, au sein des instances du Conseil de l'Europe, la question a été

posée à plusieurs reprises de l'identification des types de litiges plus particulièrement adaptés à ce mode de règlement.

La notion de «cas approprié » apparaît dans la recommandation $n^{\circ} \mathrm{R}(81) 7 \mathrm{du}$ Comité des ministres. La nécessité d'identifier les litiges à aiguiller vers des modes alternatifs a été posée à nouveau par le rapporteur général du Séminaire multilatéral, lequel ajoutait « qu'il s'agit là d'un problème complexe dont la réponse sera néanmoins capitale pour améliorer l'efficacité le mieux possible »7 . Enfin, le comité d'experts chargé de mettre au point un guide législatif des mesures visant à accroître l'efficacité de la justice (CJ-EJ ) a conclu à la nécessité de recourir à un consultant agréé indépendant, chargé d'établir un rapport "identifiant celle des situations pour lesquelles la médiation serait appropriée pour résoudre les litiges, en alternative aux procédures traditionnelles de résolution des disputes".

C'est dans le contexte de cette recherche des situations litigieuses, circonscrites au domaine des litiges civils ${ }^{8}$, auxquelles la médiation serait «appropriée », que s'inscrit la présente mission.

\section{2-3- La méthode d'évaluation}

Pour aborder cette mission, l'auteur du présent rapport disposait certes d'une expérience théorique et empirique dans le domaine des règlements non juridictionnels ${ }^{9}$, mais d'aucun moyen d'investigation empirique propre à apporter une mesure concrète des avantages apportés par une médiation dans un cas donné.

7 Jagtenberg R. W., Rapport Général au Séminaire Multilatéral sur les Modes Alternatifs de Règlement des Litiges, Strasbourg, op. cit. p. 7.

8 Définition qui exclut la médiation pénale ainsi que toutes les formes de médiation intervenant dans les rapports des usagers avec l'administration (Médiateur de la République en France, Ombudsman dans les pays nordiques, médiation et conciliation des tribunaux administratifs, etc...).

9 L'auteur du présent rapport a à son actif plusieurs travaux sur la question. Deux études empiriques ont été menées, la première sur les conciliations extra et intra-judiciaires. (Serverin E., [1985], « La conciliation et le traitement non juridictionnel des conflits privés », CERCRID, Université de SaintEtienne, multigr., 260 p.), la seconde sur le règlement amiable des accidents de la circulation (Serverin E., [1997] «Les accidents corporels de la circulation, entre transactionnel et juridictionnel », CERCRID, Saint-Etienne, Ministère de la Justice, Sept. 1997, 205 pages.). Un ouvrage a été tiré à partir des enseignements de la première recherche (Serverin E., Lascoumes P., Lambert T., [1987], « Transaction et pratiques transactionnelles », Economica). 
Revista Eletrônica de Direito Processual - REDP. Volume 15. Janeiro a Junho de 2015

Periódico Semestral da Pós-Graduação Stricto Sensu em Direito Processual da UERJ.

Patrono: José Carlos Barbosa Moreira. www.redp.com.br ISSN 1982-7636 PP 107-149

Dans ces conditions, l'expertise ne pouvait être menée que de manière conceptuelle, en construisant diverses situations types dans lesquelles la médiation peut utilement être logée. Ces situations types seront identifiées par référence principale à la législation française, en recourant aux exemples issus des autres pays chaque fois que des données de comparaison seront disponibles.

Ces situations types seront identifiées selon une double distinction : selon l'étape à laquelle intervient la médiation; selon le point de vue sous lequel l'adéquation d'un litige à la médiation peut être appréciée.

1- La première distinction revient à séparer les situations dans lesquelles la médiation intervient en amont de la saisine d'un tribunal (Première partie), et après introduction d'une instance (Deuxième partie). En effet, si on admet qu'un litige n'est pas identique selon le moment où on le saisit 10 , on doit considérer de même que la médiation diffère selon l'étape à laquelle elle intervient.

2- A chacun de ces deux moments, l'adéquation d'un litige à la médiation peut être évaluée selon trois points de vue majeurs :

- Le premier point de vue est celui de la rentabilité économique de l'intervention $\mathrm{du}$ tiers médiateur par rapport un règlement par le juge ou à un accord transactionnel entre les parties ;

- Le second point de vue conduit à évaluer les moyens juridiques par lesquels un litige est orienté préférentiellement vers la médiation ;

- Le troisième point de vue considère l'efficacité juridique des actes issus de la médiation

Ces observations seront rassemblées dans un chapitre conclusif qui proposera une synthèse des principales situations évoquées.

Première partie

Des médiations aux marches du palais

Placer la médiation au plus près du moment de la naissance des litiges, paraît a priori la démarche la plus rationnelle. Il est fréquemment soutenu que la médiation

10 . Felstiner, W., Abel, R., Sarat, A. [1980-1981], «The Emergence and Transformations of Disputes : Naming, Blaming, Claming», Law \& Society Review, 15, 631.Cette étude repère trois moments dans la naissance d'un litige : l'identification du problème (naming), l'identification de la personne responsable (blaming), et la réclamation formée devant elle (claiming). Le litige naît seulement en cas de rejet de la réclamation, ce qui suppose de placer la médiation très en amont de l'intervention d'un juge, au plus près de la personne auprès de qui la réclamation est formée. 
Revista Eletrônica de Direito Processual - REDP. Volume 15. Janeiro a Junho de 2015 Periódico Semestral da Pós-Graduação Stricto Sensu em Direito Processual da UERJ. Patrono: José Carlos Barbosa Moreira. www.redp.com.br ISSN 1982-7636 PP 107-149

présente un avantage social, en ce qu'elle permet aux parties de régler elles-mêmes leurs litiges, et en contribuant à la promotion de la paix sociale et à la conservation des relations économiques durables. Il reste à soumettre cet a priori favorable à l'épreuve de la diversité des situations litigieuses, sous le triple point de vue de l'adaptation économique, juridique, et empirique.

Chapitre 1- Des litiges économiquement adaptés à une médiation hors instance

Si on renonce au postulat commode d'une médiation qui serait en elle-même porteuse d'efficacité et de rationalité, il faut affronter en premier lieu la question des avantages économiques apportés par la médiation en termes de règlement des litiges. Pour les médiations antérieures au procès, l'avantage semblerait être toujours assuré, dans la mesure où l'arrangement obtenu permettrait d'éviter tant la durée du procès que le coût de la procédure. Les observations empiriques manquent pour vérifier cette assertion. Mais on peut indiquer les critères d'évaluation à retenir, en mettant en balance dans diverses configurations litigieuses les coûts relatifs de la médiation et du procès, et les coûts relatifs de la médiation et de la transaction.

Section 1 - Avantages comparés de la médiation et du procès

Les affirmations relatives aux avantages économiques attendus de la médiation partent de l'hypothèse d'une médiation sans frais, et d'un procès long et coûteux. Mais si on fait varier les paramètres des frais de la médiation et des frais du procès en fonction des types de litiges, la situation apparaît beaucoup plus contrastée.

\section{A-Des médiations entraînant un coût pour les parties}

Lorsque la médiation est assurée par un tiers rémunéré, désigné par les parties avant ou après le litige, il est nécessaire de comparer l'avantage qu'elle procure sur l'engagement d'une procédure. Pour cela, il faut tenir compte de la diversité des coûts du procès selon les tribunaux. Dans de nombreuses législations, le principe consiste à renvoyer les litiges de faible valeur devant des juridictions et à des procédures peu formalisées et à coût faible (notamment par dispense d'avocat), et à faire entrer les litiges dont l'enjeu est plus important dans la compétence de juridictions de niveau supérieur où les étapes du procès sont plus nombreuses et l'assistance d'un avocat obligatoire. On se doit donc de raisonner différemment selon les types de juridictions. 
Revista Eletrônica de Direito Processual - REDP. Volume 15. Janeiro a Junho de 2015 Periódico Semestral da Pós-Graduação Stricto Sensu em Direito Processual da UERJ. Patrono: José Carlos Barbosa Moreira. www.redp.com.br ISSN 1982-7636 PP 107-149

1- Les litiges entrant dans la compétence d'attribution de juridictions du premier type sont généralement ceux qui tendent au règlement de créances de faible valeur (qu'elles soient contractuelles ou statutaires), et dont le principe est souvent incontesté. Ces litiges sont traités dans de nombreux pays par des procédures rapides et unilatérales comme l'injonction de payer en France. Dans ce domaine, les avantages d'une médiation rémunérée ne sont pas certains. De plus, sa promotion viendrait contrecarrer les efforts réalisés au niveau communautaire pour rapprocher les législations en matière de procédures rapides, et dont le Conseil européen de Tampere des 15 et 16 octobre 1999 a réaffirmé la priorité, notamment pour les litiges transfrontaliers 11 .

Une médiation rémunérée devrait donc être exclue dans les litiges pour le règlement desquels existent des procédures rapides et peu coûteuses. Cela signifie notamment que de telles médiations ne doivent jamais être imposées (par voie de contrat ou autre) à des parties qui peuvent légitimement revendiquer l'accès à des juridictions rapides et peu coûteuses.

2- En revanche, dans les litiges dont la valeur, actuelle ou attendue, est élevée, la médiation peut utilement être mise en œuvre, non seulement pour éviter les dépenses d'un procès étatique (effectivement long et coûteux dans ce cas de figure), mais aussi ceux d'une procédure arbitrale lorsque cette dernière est licite. Dans ce dernier cas, qui concerne les litiges entre professionnels, il peut être en effet préférable d'introduire une procédure de négociation sous l'égide d'un tiers plutôt que de devoir mettre en place un arbitrage. Les parties y trouveraient l'intérêt de bénéficier d'une phase de négociation, sans risquer d'engager définitivement leurs droits, et surtout sans avoir à financer la mise en place d'un véritable tribunal arbitral.

Il en résulte que la médiation rémunérée anticipée peut être économiquement adaptée aux litiges dont la valeur, actuelle ou attendue, est élevée, et parmi ces derniers, à ceux qui sont arbitrables.

11 Dans ses conclusions, le Conseil européen demandait au Conseil d'établir, sur la base de propositions de la Commission, "des règles de procédures spéciales communes en vue de simplifier et d'accélérer le règlement des litiges transfrontaliers concernant les demandes de faible importance en matière civile et commerciale ainsi que les créances alimentaires et les créances certaines" (§. 30). A la Commission, il était demandé de faire une proposition en matière de reconnaissance mutuelle des décisions judiciaires et "tendant à supprimer les procédures intermédiaires pour les droits concernant les demandes de faible importance en matière civile" (§. 34). 
Revista Eletrônica de Direito Processual - REDP. Volume 15. Janeiro a Junho de 2015 Periódico Semestral da Pós-Graduação Stricto Sensu em Direito Processual da UERJ. Patrono: José Carlos Barbosa Moreira. www.redp.com.br ISSN 1982-7636 PP 107-149

$B$-Des médiations sans coût pour les parties

Il y aura médiation sans coût pour les parties lorsque la collectivité met à leur disposition des médiateurs (qu'elle rémunère ou non), et qui disposent, selon les cas, d'une compétence spéciale ou d'une compétence générale.

En nous en tenant à une simple balance coût/ avantage pour les parties (c'est-àdire sans tenir compte de la dépense publique induite par la mise en place de telles médiations), l'intérêt de la médiation varie cette fois selon la probabilité du procès.

1- Si le litige en cause présente des caractéristiques telles qu'on peut penser que le demandeur n'aurait pas intenté de procès (faible valeur du litige, aversion pour le risque, disproportion entre les moyens des adversaires), le recours à un médiateur bénévole est économiquement avantageux. Cet avantage ne découle pas de l'économie des frais d'une procédure, puisque celle-ci n'aurait pas été engagée. Il réside dans l'octroi d'une chance d'obtenir un résultat économiquement favorable dans une situation de déséquilibre entre les parties.

La médiation doit être encouragée dans les litiges où les demandeurs potentiels sont peu actifs, dans la perspective d'une mise en auvre effective de leurs droits. C'est bien en ce sens que vont les travaux de la Communauté européenne en matière de modes alternatifs de règlement des litiges et notamment, la Recommandation du 30 mars 1998 sur les principes applicables aux instances pour le règlement extrajuridictionnel des litiges des consommateurs.

2- Si, au contraire, la probabilité que le demandeur intente une procédure est élevée, notamment en raison de contraintes d'action pesant sur lui (par exemple, un gestionnaire d'immeubles qui doit recouvrer des créances de loyer pour le compte de ses clients), l'intervention d'un médiateur, même bénévole, ne se justifie pas, dans la mesure où cette intervention ne pourrait que retarder le moment du recouvrement. Cette situation est celle des contentieux de masse (et souvent de faible valeur) comme les paiements de créances certaines. Mais dans ce secteur, les créanciers peuvent également avoir intérêt à obtenir un règlement amiable, pour éviter d'avoir à introduire une procédure, ou à recourir aux services d'officines de recouvrement. La mise à disposition d'une médiation sans coût pour les parties peut alors être utile, comme substitut de ces voies plus ou moins coercitives. En tout état de cause, une telle médiation ne peut être que proposée, sauf si les intérêts de la partie débitrice de l'obligation sont considérés 
Revista Eletrônica de Direito Processual - REDP. Volume 15. Janeiro a Junho de 2015 Periódico Semestral da Pós-Graduação Stricto Sensu em Direito Processual da UERJ. Patrono: José Carlos Barbosa Moreira. www.redp.com.br ISSN 1982-7636 PP 107-149

comme particulièrement dignes de protection, auquel cas la médiation peut constituer un passage obligé pour l'accès au tribunal.

Une médiation anticipée sans frais pour les parties peut être proposée dans le secteur des impayés, et imposée s'il apparaît que les intérêts des défendeurs méritent de faire l'objet d'une protection particulière.

Section 2 - Avantages comparés de la médiation et de la négociation transactionnelle

La médiation n'est pas le seul procédé permettant d'obtenir un règlement anticipé antérieur à la saisine du tribunal. La procédure transactionnelle menée directement entre les parties, avec ou sans intervention d'un conseil, permet d'aboutir au même résultat: la conclusion d'un accord transactionnel. A priori, le processus transactionnel ne génère pas de « coûts de transaction » (pour reprendre la formulation des économistes), dès lors que les parties au litige sont identifiées, et acceptent de négocier. Mais un processus transactionnel n'est efficace que si les parties sont prêtes à négocier. En cas de réticence de l'une d'elles (notamment, de celle qui n'a aucun intérêt à faire une offre), le recours à un médiateur peut être un moyen de l'inciter à entrer dans un processus de négociation. Il faut donc être attentif aux circonstances qui favorisent cette inclination à négocier.

\section{A-Médiations et processus transactionnel spontané}

La transaction se définit dans la plupart des droits continentaux comme un «contrat par lequel les parties terminent une contestation née ou préviennent une contestation à naître » (art. 2044 du code civil français). Cette dernière forme de règlement est toujours sans frais pour les parties, puisqu'elle est directement assurée par elles. Elle est préférable à tout type de médiation dès lors que les parties sont en mesure de transiger, et trouvent un intérêt réciproque à la négociation, ce qui est la situation de loin la plus fréquente. Il est une évidence de la vie quotidienne que la plupart des litiges s'y résolvent de manière transactionnelle, encore que les parties n'aient pas eu en vue l'accomplissement d'un tel contrat. Elles peuvent être menées soit directement entre les parties, soit avec l'aide de conseils qui peuvent jouer un véritable rôle de médiateur. C'est bien du reste ce que semble considérer la loi française du 18 décembre 1998 sur «l'accès au droit et la résolution amiable des conflits » modifiant la loi du 10 juillet 1991, qui a étendu le bénéfice de l'aide juridictionnelle aux actes accomplis par les 
Revista Eletrônica de Direito Processual - REDP. Volume 15. Janeiro a Junho de 2015 Periódico Semestral da Pós-Graduação Stricto Sensu em Direito Processual da UERJ. Patrono: José Carlos Barbosa Moreira. www.redp.com.br ISSN 1982-7636 PP 107-149 avocats en vue de « parvenir à une transaction avant l'introduction de l'instance (art. 10 al. 2 nouveau ). Ces transactions reviennent parfois au contentieux, ce qui permet de prendre conscience (sinon de mesurer l'importance) de la vigueur de ce contrat. En France, les transactions consécutives à la rupture d'un contrat de travail alimentent une jurisprudence très dense, qui témoigne de l'ampleur de la pratique de négociation dans ce secteur, menée à l'ombre des directions des ressources humaines et avec l'appui des avocats spécialisés.

Cependant, les parties peuvent n'avoir aucun intérêt à entrer dans un processus de négociation. Il peut alors être opportun de les y inciter par la mise en place de procédures obligatoires.

\section{B-Médiation et processus transactionnel obligatoire}

Le droit français offre un exemple particulièrement probant de procédure transactionnelle obligatoire dans le domaines des accidents corporels de la circulation. La loi du 5 juillet 1985 a mis à la charge de l'assureur une offre obligatoire d'indemnité, qui doit être présentée à la victime qui a subi une atteinte à la personne (art. L.211-8 du code des assurances). L'obligation pesant sur l'assureur peut s'analyser comme une contrainte d'entrer dans un processus de négociation avec la victime, même si celle-ci $n$ 'a formé aucune réclamation ${ }^{12}$. L'entrée dans la phase de proposition est sans risque pour la victime, qui dispose tout au long du processus d'offre de l'option entre l'acceptation d'un règlement conventionnel, et la demande de fixation judiciaire de l'indemnité. Si le processus transactionnel s'effectue hors de tout procès, le juge peut néanmoins être amené à intervenir à des titres divers: pour parfaire certaines transactions (celles concernant les mineurs), pour contrôler les propositions de l'assureur en cours de procédure, et surtout, pour contrôler a posteriori la régularité de la procédure d'offre et l'équilibre des transactions conclues (nullités spéciales).

Ce type de négociation ne fait nullement appel à des tiers, et ne relève donc pas de la médiation. Mais en palliant les insuffisances de la volonté de négocier, le dispositif permet tout à la fois d'assurer une bonne application d'une loi qui reconnaît un droit à

12 Cette obligation constitue une dérogation au principe général posé par l'article L.124-1 du code des assurances selon lequel, "dans les assurances de responsabilité, l'assureur n'est tenu que si, à la suite du fait dommageable prévu au contrat, une réclamation amiable ou judiciaire est faite à l'assuré par le tiers lésé". 
Revista Eletrônica de Direito Processual - REDP. Volume 15. Janeiro a Junho de 2015 Periódico Semestral da Pós-Graduação Stricto Sensu em Direito Processual da UERJ. Patrono: José Carlos Barbosa Moreira. www.redp.com.br ISSN 1982-7636 PP 107-149

indemnité aux victimes d'accident corporel, et un recours raisonné au tribunal, comme nous avons pu nous-mêmes le constater sur le terrain 13 .

Dans les litiges dont le règlement négocié dépend de la bonne volonté d'une partie économiquement puissante, le procédé le plus efficace consiste à contraindre la partie dominante à entrer dans un processus d'offre directe, plutôt que d'instaurer une médiation qui ajoute un intermédiaire et diffère le moment du règlement.

Chapitre 2- Des litiges juridiquement adaptés à une orientation vers des médiations hors instance

Si l'aménagement de l'accès aux tribunaux est un devoir des Etats, dont le respect est garanti par des instruments juridiques internationaux, l'institution d'une procédure de médiation hors des tribunaux est abandonnée à leur initiative et à celle des personnes privées. Deux voies permettent d'orienter les litiges vers une médiation: les réglementations étatiques qui créent des instances à cet effet; les parties intéressées elles-mêmes de manière unilatérale ou conventionnelle.

Section 1 - Les litiges orientés vers la médiation par la législation des Etats

L'autorité législative peut promouvoir une médiation hors de toute instance, qui remplit une double fonction: l'évitement du procès, et la mise en œuvre du droit. En l'absence d'un principe général du droit à la médiation, cette initiative tend à se faire au coup par coup, en fonction de l'intérêt qui est porté à telle ou telle catégorie de litige.

Les matières litigieuses concernées seront tantôt largement définies, tantôt étroitement spécialisées.

\section{A- Le renvoi à la médiation de litiges non définis}

Le législateur peut instaurer des médiateurs «généralistes » pour le règlement de litiges à définition large. L'institution française des «conciliateurs de justice » en est le prototype. Créés par un décret du 28 mars 1978 plusieurs fois modifié, les conciliateurs (devenus conciliateurs de justice en 1996), ont «pour mission de faciliter, en dehors de toute procédure judiciaire, le règlement amiable des différends portant sur des droits dont les intéressés ont la libre disposition», et peuvent être saisis par toute personne

13 Voir notre étude précitée, «L'accident corporel de la circulation, entre transactionnel et juridictionnel » [1997] 
Revista Eletrônica de Direito Processual - REDP. Volume 15. Janeiro a Junho de 2015 Periódico Semestral da Pós-Graduação Stricto Sensu em Direito Processual da UERJ. Patrono: José Carlos Barbosa Moreira. www.redp.com.br ISSN 1982-7636 PP 107-149 physique ou morale ou par les autorités judiciaires. La sphère de compétence de ces conciliateurs a toujours été étendue, après une tentative de courte durée pour créer une spécialisation en matière de consommation (entre le décret du 25 février 1993 et le décret du 22 juillet 1996). Quant aux compétences requises des personnes, elles oscillent entre les qualités personnelles et la compétence juridique. Les conciliateurs ont d'abord été nommés au vu de «qualités humaines qui les rendront aptes à exercer ces fonctions» ${ }^{14}$, puis le décret du 25 février 1993 a exigé qu'ils disposent d'une expérience d'au moins cinq années en matière juridique, réduite à trois par le décret du 13 décembre 1996. La circulaire d'application de ce dernier texte $\left(n^{\circ} 67\right.$ du 30 septembre 1997), explique cette réduction de la durée de l'expérience professionnelle par la préférence donnée à des critères subjectifs, comme « le sens de l'écoute, sens de l'analyse, goût des contacts humains». Ces conciliateurs se conçoivent eux-mêmes comme une instance d'écoute plus que de consultation, et manifestent une aversion toute particulière envers toute forme de production écrite 15 .

Ces hésitations témoignent surtout de la difficulté à trouver une place à des conciliateurs dont les activités tendent à se dérouler de plus en plus à l'ombre des tribunaux. En témoigne la loi du 18 décembre 1998 qui fait des maisons de justice le lieu d'accueil de l'activité des conciliateurs agissant sur mandat du juge ${ }^{16}$.

L'activité de ces médiateurs apparait singulièrement réduite si l'on considère l'ampleur de leur spectre d'action. Les statistiques officielles font état de 85.451 litiges traités par les 1614 conciliateurs en poste en 1998, soit une moyenne de 53 affaires par conciliateur et par an. Cet effectif représente $7,7 \%$ des affaires nouvelles reçues au cours de la même année par les 654 tribunaux statuant au premier degré en matière civile (1.101.880 affaires pour les tribunaux d'instance et de grande instance) ${ }^{17}$.

14 Instruction générale du 26 avril 1978 relative au décret du 28 mars 1978 sur les conciliateurs.

15 Depuis le décret du 13 décembre 1996, l'établissement d'un constat d'accord a été rendu facultatif, à la demande de nombreux conciliateurs. Et il n'est en aucun cas question pour le conciliateur de rendre un avis, contrairement aux commissions de conciliation spécialisées.

16 L'article L. 7-12-1-1 nouveau du Code de l'organisation Judiciaire prévoit "qu'il peut être institué des maisons de justice et du droit, placées sous l'autorité des chefs du tribunal de grande instance dans le ressort duquel elles sont situées", leurs modalités de création et de fonctionnement restant à déterminer par décret en Conseil d'Etat. Ces maisons ont pour finalité "d'assurer une présence judiciaire de proximité et concourent à la prévention de la délinquance, à l'aide aux victimes et à l'accès au droit, ainsi qu'à l'application des mesures alternatives de traitement pénal et les actions tendant à la résolution amiable des litiges $»$,

17 Annuaire statistique de la justice, éd. 2000, La Documentation Française, p. 279. 
Revista Eletrônica de Direito Processual - REDP. Volume 15. Janeiro a Junho de 2015 Periódico Semestral da Pós-Graduação Stricto Sensu em Direito Processual da UERJ. Patrono: José Carlos Barbosa Moreira. www.redp.com.br ISSN 1982-7636 PP 107-149

Ce faible recours au conciliateur de justice résulte sans doute de la concurrence exercée par les diverses voies de règlement ouvertes aux plaideurs dans un pays comme la France. L'utilité de médiateurs bénévoles non spécialistes ne peut donc être que marginale, et dépendra des moyens dont disposent les Pays concernés de proposer des médiations plus diversifiées. La médiation généraliste ne devrait être proposée que de manière circonstanciée, en considération des particularités du Pays concerné.

\section{$B$ - L'institution de médiations spécialisées}

Nombre de pays établissent, à côté des tribunaux, des organismes qui sont chargés de régler à l'amiable les litiges spécialisés, le plus souvent avec la volonté affichée d'en faire un instrument d'application d'une législation dans des secteurs où l'action des justiciables reste faible et dispersée. Cet argumentaire a du reste étayé un des considérants de la Commission européenne dans sa recommandation du 30 mars 1998 relative aux principes applicables aux organes responsables pour la résolution extrajudiciaire des litiges. Selon la Commission, «la majorité des litiges de consommation, de par leur nature, se caractérisent par une disproportion entre l'enjeu économique de l'affaire et le coût de leur règlement judiciaire (...) les difficultés éventuelles liées aux procédures judiciaires peuvent, notamment dans le cas des conflits transfrontaliers, dissuader le consommateur de faire valoir effectivement ses droits». ${ }^{18}$. Aujourd'hui, les Etats tendent à faire des médiations spécialisées un mode spécifique d'application de leurs législations.

1- C'est le cas pour la Finlande des «Municipal Consumer Advisors », mis en place en 1978 au niveau local, avec pour objectif de fournir des services d'information et d'aide aux consommateurs. Ces consultants remplissent également une fonction de médiation, qu'ils doivent mener, selon les travaux préparatoires de la loi, « de manière impartiale et dans le but de défendre les droits substantiels des consommateurs $» 19$.

- En France, les instances de conciliation spécialisées se sont multipliées depuis les vingt dernières années, dans les domaines les plus divers, et à destination des publics les plus variés. On ne peut donner ici plus que des exemples de ce qui tend à devenir un procédé général de traitement des litiges spécialisés.

18 Recommandation de la Commission n $n^{\circ}$ 98/257/CE, 30 mars 1998, JOCE, vol. L115, 17 avril 1998, p. 31.

19 Une enquête menée en 1998 sur les actions de ces conseillers montre que $15 \%$ des plaintes déférées font l'objet d'une médiation avec le professionnel, et que les $4 / 5^{\circ}$ donnent lieu à un accord, dont les $9 / 10^{\circ}$ à l'avantage du demandeur. Teittinen S.,[1999], « Municipal Consumer Advisors and Consumer Complaints », National Research Institute of Legal Policy, Publication n ${ }^{\circ} 158$, Helsinki. 
Revista Eletrônica de Direito Processual - REDP. Volume 15. Janeiro a Junho de 2015 Periódico Semestral da Pós-Graduação Stricto Sensu em Direito Processual da UERJ. Patrono: José Carlos Barbosa Moreira. www.redp.com.br ISSN 1982-7636 PP 107-149

- Parmi les litiges privés à caractère professionnel, on citera les procédures de conciliation et de médiation organisées dans le domaine des conflits collectifs du travail (art. L. 523-1 à L. 524-8 du code du travail), ), la commission paritaire de conciliation compétente en matière de litige sur l'indemnisation d'une invention de salarié (art. L.615-21 et s. Code de la propriété intellectuelle), la commission de conciliation des litiges «pouvant survenir entre les acheteurs de lait et l'office national interprofessionnel du lait et des produits laitiers (art. L. 632-2 du code rural) », la conciliation réalisée par l'Autorité de régulation des télécommunications» (art. L.36-9 nouveau du Code des Postes et télécommunications), les médiateurs «chargés de la résolution des différends relatifs à l'octroi de l'autorisation de retransmission par câble» (Titre II du Livre III du Code de la propriété intellectuelle).

- Au titre des litiges commerciaux, on retiendra la très importante commission départementale de conciliation compétente en matière de révision ou de renouvellement des loyers commerciaux (décret du 30 septembre 1953 et loi du 5 janvier 1988). Cette commission paritaire peut être saisie par le bailleur ou le preneur avant toute procédure juridictionnelle 20 , et les services (gratuits) de médiation qu'elle offre visent à « concilier bailleurs et locataires tout en recherchant un compromis juste dans le cadre des dispositions législatives et réglementaires en vigueur $»^{21}$.

- C'est dans les litiges mettant en cause des non-professionnels que l'on trouve aujourd'hui les procédures de conciliation d'origine étatique les plus nombreuses. La matière de la consommation connaît de longue date des procédures d'intermédiation (boîte postale 5000, puis commissions de règlement des litiges de consommation, et conciliateurs spécialisés en matière de consommation jusqu'au décret du 22 juillet 1996). La révision des loyers des baux d'habitation donne lieu à saisine préalable d'une commission départementale de conciliation (art. 20 de la loi du 6 juillet 1989). Le surendettement des ménages est traité avant toute saisine d'un tribunal par une commission départementale chargée d'élaborer un plan de redressement conventionnel entre le débiteur et ses créanciers (art. R.331-16 du Code de la consommation). Le domaine de la santé est couvert par la nouvelle commission de conciliation mise en place conciliation mise en place par le décret du 2 novembre 1998 pris en application de

20 La Cour de cassation a considéré que la saisine de cette commission était facultative, mais qu'en cas de saisine, le juge devait surseoir à statuer jusqu'à remise de l'avis (art. 23-6-1 de la loi du 30 septembre 1953).

$21 \quad$ Circulaire du 3 août 1988, JO du 25 septembre 1988, p. 12198. 
Revista Eletrônica de Direito Processual - REDP. Volume 15. Janeiro a Junho de 2015

Periódico Semestral da Pós-Graduação Stricto Sensu em Direito Processual da UERJ.

Patrono: José Carlos Barbosa Moreira. www.redp.com.br ISSN 1982-7636 PP 107-149

l'ordonnance 96-346 du 24 avril 1996 (art. L.1112-3 du nouveau Code de la santé

publique), et destinée à «assister et orienter toute personne qui s'estime victime d'un préjudice du fait de l'activité de l'établissement et de lui indiquer les voies de conciliation et de recours dont elle dispose». L'accès au logement des personnes défavorisées est étudié par une «commission de médiation paritaire » (art. 56-1 du Code de la construction et de l'habitat).

2- La diversité et le nombre de ces procédures rendent impossible une synthèse de leur composition, de leur compétence et de la procédure suivie. Mais. quel que soit le domaine couvert, on peut noter que ces instances de médiation ont en commun quatre traits essentiels:

- l'accès en est gratuit pour les parties,

- elles sont composées de manière paritaire, de façon à garantir la représentation de chacune des catégories d'intérêts en présence dans un litige déterminé,

- elles disposent d'une compétence d'attribution strictement délimitée par les règles dont elles ont vocation à garantir l'application. 22

- elles ont un rôle actif dans la recherche de solutions, notamment par un pouvoir de proposition matérialisé par des écrits (avis, recommandations, rédaction de projets d'accords).

On aura reconnu là les critères que la Commission européenne a retenus pour définir les missions de médiation des organes intervenant en matière de litiges de consommation. Cette recommandation introduit en effet une distinction dans les positions des médiateurs selon «qu'elles se limitent à une simple tentative de rapprocher les parties pour les convaincre de trouver une solution ou qu'elles constituent de véritables décisions, pouvant, selon les cas, être à effet contraignant pour les parties ou se résumer à de simples recommandations ou à des propositions de transactions qui doivent être acceptées par les parties ». Si notre définition de la médiation comme tiers intervenant à titre amiable est plus restrictive que celle de la Commission (les instances dont les décisions sont obligatoires constituent en effet un tribunal arbitral), on partagera la préoccupation de donner au tiers médiateur un rôle

22 Hors des limites de cette compétence, l'intervention des instances de médiation est prohibée. On peut prendre l'exemple de la procédure de conciliation et d'arbitrage prévue par la loi du 10 juillet 1975 relative à l'organisation interprofessionnelle agricole. Cette procédure n'est pas applicable aux actions en recouvrement des cotisations professionnelles, lesquelles peuvent être diligentées après une simple mise en demeure. Pour une application de cette clause, voir Cour de cassation, Première chambre civile, 23 février 1994, pourvoi n 92-14.111, Lamy n 321 . 
Revista Eletrônica de Direito Processual - REDP. Volume 15. Janeiro a Junho de 2015 Periódico Semestral da Pós-Graduação Stricto Sensu em Direito Processual da UERJ. Patrono: José Carlos Barbosa Moreira. www.redp.com.br ISSN 1982-7636 PP 107-149 actif dans le règlement du litige matérialisé par la production d'un écrit, distinct de celui que les parties auront pu produire comme instrumentum de leur accord.

Ce type de médiation représente une forme très élaborée de règlement non juridictionnel des litiges. L'intérêt de ce genre de procédure est le plus grand dans les domaines où s'affrontent des parties de force inégale, impliquant notamment des usagers et des consommateurs. En effet, l'instauration de médiations spécialisées (qu'elles soient obligatoires ou facultatives) crée une contrainte pour la partie dominante, qui se voit amenée à participer à une négociation qu'un locataire, un consommateur ou un patient n'auraient pas eu les moyens d'imposer.

Les Etats devraient être encouragés à mettre gratuitement à disposition des usagers des commissions de médiation spécialisées notamment partout où les parties en présence sont de force inégale, dans des conditions qui garantissent l'équilibre des intérêts et l'application du droit en cause dans le litige.

Section 2- Les litiges orientés vers la médiation par la convention des parties

Les parties en présence peuvent elles-mêmes prévoir le recours à un tiers médiateur en cas de litige, soit sur initiative de l'une d'entre elles, soit par le jeu d'une clause de règlement amiable insérée au contrat.

\section{A-L'offre unilatérale de médiation}

Il arrive que des «services de médiation» soient mis en place de manière unilatérale par des professionnels qui y trouvent l'avantage de garder la maîtrise du règlement des litiges dans le cadre des contrats dont ils ont l'usage. Certains de ces «services» ont été pris en application des recommandations de la Commission européenne sur les codes de conduite pour la protection des consommateurs. En France, la Fédération Européenne de la vente directe a ainsi mis en place un Code de conduite européen, en usage dans quinze pays européens, et qui prévoit un dispositif de médiation complet comprenant un Conseil de médiation et une Commission paritaire de Médiation 23 . Certes, ces instances sont composées de manière paritaire (représentants des usagers et de la profession). Mais comme leur nom l'indique, elles sont mises en place à titre de «services » par le Syndicat de la vente directe, ce qui, en l'absence de véritable tiers intervenant, permet de douter de l'adéquation du terme de « médiation ».

23 Pour une présentation des activités de ces services, voir le rapport présenté par le délégué général de ce syndicat, Philippe Dailey, lors du Séminaire Multilatéral, doc. SEM/ADR (99) 16F. 
Revista Eletrônica de Direito Processual - REDP. Volume 15. Janeiro a Junho de 2015 Periódico Semestral da Pós-Graduação Stricto Sensu em Direito Processual da UERJ. Patrono: José Carlos Barbosa Moreira. www.redp.com.br ISSN 1982-7636 PP 107-149

Le recours à ces services doit rester en tout état de cause une simple faculté pour les parties.

\section{$B$ - Des clauses de médiation}

- Les clauses de médiation/ conciliation présentent en revanche une contrainte pour les parties. Dans leurs conventions en effet, les parties peuvent toujours convenir de désigner un médiateur en cas de litiges entre elles. Ces clauses de règlement amiable, parce qu'elles ne mettent pas en jeu le fond du droit, ne sont pas soumises aux restrictions introduites par les législations aux clauses tendant à constituer un tribunal arbitral (clauses compromissoires). En France, selon l'article 2061 du Code civil, la nullité de la clause compromissoire est de principe, sauf autorisation expresse de la loi, autorisation qui n'est accordée que pour les litiges entre professionnels. ${ }^{24}$ A priori donc, leur insertion dans les contrats est licite, ouvrant ainsi un champ infini d'action aux médiateurs privés, avec toutes les formes de composition de qualification et de rémunération que peuvent imaginer les parties.

Cependant, on peut discuter de la licéité de ces clauses lorsqu'elles sont imposées, et qu'elles pour ont pour but ou pour effet de limiter le droit de recours d'un individu à un tribunal. L'engagement contractuel de recourir préalablement à un médiateur avant d'intenter une action en justice peut en effet constituer une forme détournée de limitation au droit d'agir en justice, notamment lorsqu'il entraîne un coût pour la partie demanderesse. C'est dire que ces clauses doivent être examinées avec attention dans les différents droits positifs. Quelques indications peuvent être données à cet égard en prenant appui sur le droit français et européen.

- Dans les rapports entre consommateurs et professionnels, la Commission des clauses abusives française a pris le 30 janvier 1979 une recommandation entièrement consacrée aux clauses relatives aux recours en justice. Cette recommandation prohibe les clauses constituant une renonciation anticipée au droit d'agir en justice, et notamment celles par lesquelles « un non-professionnel ou consommateur s'oblige à recourir à la seule voie amiable pour régler un litige éventuel» ou le « recours à un arbitrage $»^{25}$.

24 Article $631 \mathrm{du}$ code de commerce. Le maintien de la prohibition de principe des clauses compromissoires à l'égard des non commerçants paraît tout à fait indispensable pour éviter les dérives consistant à imposer l'arbitrage à de parties qui n'en ont ni les moyens ni la pratique.

25 CCA nº79-02 du 30 janvier 1979, BOCC 13 juin 1979. 
Revista Eletrônica de Direito Processual - REDP. Volume 15. Janeiro a Junho de 2015 Periódico Semestral da Pós-Graduação Stricto Sensu em Direito Processual da UERJ. Patrono: José Carlos Barbosa Moreira. www.redp.com.br ISSN 1982-7636 PP 107-149

- Le droit communautaire a réglementé ce type de clauses dans des termes voisins de ceux de la Commission des clauses abusives. La Directive CEE du 5 avril 1993 concernant les clauses abusives dans les contrats conclus avec les consommateurs fait figurer dans l'annexe indiquant les clauses considérées comme telles un paragraphe q), visant les clauses qui ont pour objet ou pour effet «de supprimer ou d'entraver l'exercice d'actions en justice ou des voies de recours par le consommateur, notamment en obligeant le consommateur à saisir exclusivement une juridiction d'arbitrage non couverte par des dispositions légales ». Si ce texte vise moins clairement la voie amiable, l'emploi de l'adverbe notamment signifie que la clause compromissoire n'est pas la seule prohibée, et que le caractère abusif peut être reconnu à toute clause qui constitue une entrave à l'exercice d'un recours judiciaire.

- La jurisprudence française donne des exemples de plus en plus nombreux de clauses de ce type dans les contrats les plus divers. Lorsqu'elles sont invoquées devant les tribunaux, ces clauses fonctionnent comme des fins de non-recevoir à l'action du demandeur qui entend poursuivre ses droits en justice, aussi longtemps que la tentative de conciliation prévue par la convention n'aura pas eu lieu. Certes, il s'agit d'un simple différé dans le recours au tribunal. Mais la procédure s'en trouve considérablement alourdie, et on voit poindre le danger d'une renonciation à un des droits fondamentaux de la personne, celui d'agir en justice. Le traitement de ces clauses par Cour de cassation varie selon les chambres, et il semble que la distinction entre professionnels et non professionnels fasse le partage entre le licite et l'illicite, à l'instar des clauses compromissoires.

Ainsi, dans le droit du travail français, et depuis de très nombreuses années, la Chambre sociale de la Cour de cassation écarte les clauses qui tendent à éluder la compétence du juge du travail. Selon cette chambre, les conventions collectives ne peuvent utilement prévoir la saisine d'une commission de conciliation préalablement à l'engagement d'une instance, en raison de la «compétence d'ordre public de la juridiction prud'homale devant un tribunal »26.

26 Cour de cassation Civile, Section sociale, 27 octobre 1959, pourvoi n $58-40-109$. Pour une application récente, v. Cour de cassation, Chambre sociale, 26 janvier 1994, pourvoi n $91-40.464$, Lamy $n^{\circ} 387$. Dans ce dernier arrêt, c'est la convention collective nationale des personnels des organismes de sécurité sociale qui avait prévu en cas de litige la saisine d'une commission paritaire. La Cour a affirmé que la création de tels organismes de conciliation «ne saurait faire obstacle à la saisine directe de la juridiction prud'homale par les intéressés ». 
Revista Eletrônica de Direito Processual - REDP. Volume 15. Janeiro a Junho de 2015 Periódico Semestral da Pós-Graduação Stricto Sensu em Direito Processual da UERJ. Patrono: José Carlos Barbosa Moreira. www.redp.com.br ISSN 1982-7636 PP 107-149

En revanche, ces clauses sont généralement déclarées valables dans les relations entre professionnels 27 . Elles ne font pas l'objet de traitement particulier, et sont interprétées comme toute autre clause contractuelle, notamment au regard de l'effet relatif des contrats 28 ou du régime des renonciations tacites 29.

En conclusion, on rappellera d'abord que les clauses de médiation figurant dans un contrat ont force obligatoire pour les parties. Elles doivent donc être traitées selon les dispositions pertinentes du droit des contrats en ce qui concerne la mise en place de la procédure de médiation et le caractère obligatoire des accords qui peuvent être conclus. Cependant, à l'égard des non professionnels, ces clauses ne peuvent avoir pour effet de faire obstacle à la saisine d'un tribunal.

Chapitre 3- Des litiges efficacement réglés par une médiation hors instance

Un des arguments les plus fréquemment avancés au profit de la médiation est qu'elle permet de favoriser l'échange entre les parties, et d'éviter de rompre les liens entre des personnes qui sont amenées à poursuivre des relations dans le temps, pour des raisons de proximité géographique, professionnelle ou familiale.

Cet argument est utilisé également pour réfuter les objections élevées contre les médiations payantes: la dépense effectuée serait compensée par la dépense évitée en litiges ultérieurs. En bref, la médiation remplirait une fonction d'évitement des litiges par le rétablissement de la paix sociale.

Si on ne peut qu'adhérer au principe de préférence de la paix sociale au conflit permanent, il est bien difficile d'apporter la preuve qu'une médiation est à même de parvenir au rétablissement de relations pacifiées entre les parties. Les vertus prêtées à la médiation étant purement déclaratives, on se bornera à envisager l'efficacité des actes

27 Dans le sens de la licéité d'une clause de conciliation préalable dans un contrat conclu entre une clinique et des médecins, v; Cour de cassation, Chambre commerciale, 28 nov. 1995, pourvoi n9412.285, Lamy $\mathrm{n}^{\circ} 1972$, confirmé par Cour de cassation, Deuxième Chambre civile, 6 juillet 2000, pourvoi $n^{\circ} 98-17.827$, Lamy $n^{\circ} 803$; à propos d'une clause impliquant une demande d'avis avant toute procédure judiciaire dans un contrat entre une société et un bureau d'études Cour de cassation, Troisième chambre civile, 5 juin 1996 pourvoi n $94-17.793$ et 94-17.794, Lamy n 1047 et 1048 .

28 Cour de cassation, Première chambre civile, $1^{\text {er }}$ décembre 1999, pourvoi $n^{\circ} 97-18.577$, Lamy $^{\circ}$ 1924.

29 Cour de cassation, Chambre commerciale, 8 juin 1999, pourvoi $n^{\circ} 97-17.464$, Lamy ${ }^{\circ} 1193$, Bulletin civil. 
Revista Eletrônica de Direito Processual - REDP. Volume 15. Janeiro a Junho de 2015 Periódico Semestral da Pós-Graduação Stricto Sensu em Direito Processual da UERJ. Patrono: José Carlos Barbosa Moreira. www.redp.com.br ISSN 1982-7636 PP 107-149

sous un angle strictement juridique. On se demandera d'abord dans quelle mesure la médiation garantit l'efficacité de l'allocation des droits, et de quelle sorte d'efficacité sont dotés les actes qui en sont issus.

Section 1- Des droits efficacement préservés par une médiation hors instance

On peut se demander si les droits engagés dans les litiges sont mieux préservés par le recours à une médiation, et dans quelle mesure le médiateur peut être tenu pour responsable d'avoir favorisé un accord déséquilibré.

\section{A- La garantie de l'équilibre des droits}

Il faut affirmer d'emblée que, d'un point de vue strictement juridique, la seule intervention d'un tiers médiateur n'est pas garante de l'équilibre de l'accord conclu. La transaction conclue sous son égide ne bénéficie d'aucune présomption de régularité, même si ce dernier appose sa signature sur l'acte. Il en résulte que si cet accord semble après coup inéquitable à l'une des parties, cette dernière peut en demander la nullité pour des causes variées, parmi lesquelles l'absence de réciprocité des concessions joue un rôle essentiel. Pour illustrer ce défaut de garantie, on peut prendre l'exemple fourni par un récent arrêt de la Première chambre civile de la cour de cassation, statuant sur la validité d'un procès-verbal de conciliation conclu entre des médecins sous l'égide du président de la section locale de l'Ordre national des médecins. La Cour a considéré que ce procès-verbal ne pouvait être opposé à une partie qu'après vérification de l'existence de concessions réciproques des parties 30 .

La présence d'un médiateur lors de la conclusion d'une transaction ne constitue pas une garantie de validité du contrat conclu sous son égide. En conséquence, la nullité peut toujours être demandée pour des causes tirées des règles générales de validité des conventions.

\section{$B$ - La responsabilité du médiateur dans l'équilibre de la convention}

Si l'intervention d'un médiateur n'a pas d'incidence directe sur la validité des accords, la manière dont il réalise sa mission peut être reprochable. Notamment, son intervention peut avoir été partiale, ou inadéquate, de sorte qu'une partie peut estimer avoir de ce fait subi un dommage. Certes, la partie qui s'estime lésée peut demander la

30 Cour de cassation, Deuxième Chambre civile, 3 mai 2000, pourvoi $n^{\circ} 98-12.819$, Lamy $n^{\circ} 769$, Bulletin civil. 
Revista Eletrônica de Direito Processual - REDP. Volume 15. Janeiro a Junho de 2015 Periódico Semestral da Pós-Graduação Stricto Sensu em Direito Processual da UERJ. Patrono: José Carlos Barbosa Moreira. www.redp.com.br ISSN 1982-7636 PP 107-149

nullité de l'acte conclu dans ces conditions. Mais elle peut aussi avoir intérêt à engager la responsabilité du médiateur s'il apparaît que l'attitude de ce dernier a été à l'origine $\mathrm{du}$ vice du contrat. Un tel recours doit toujours être ouvert aux parties, dans des conditions qui varieront selon la qualité du tiers intervenant.

- Si le médiateur est un professionnel indépendant, et surtout si cette activité est à but lucratif et s'effectue à grande échelle comme c'est le cas en Grande-Bretagne ${ }^{31}$, il doit en tout état de cause assurer son activité pour répondre de ses fautes éventuelles. En effet, son activité s'apparente à une prestation de service, dont la qualité doit pouvoir être évaluée à l'égal de toutes les autres prestations. Dans ce cas, la réclamation sera formée auprès de l'assureur de responsabilité du médiateur.

- Lorsque le médiateur intervient au titre d'une activité réglementée par l'Etat, on peut considérer qu'il participe à un service public, et ses fautes éventuelles peuvent engager la responsabilité de l'Etat.

Mais en tout état de cause, compte tenu de la portée juridique des accords, il importe que le médiateur soit en mesure de répondre des conséquences de son intervention.

Section 2 - L'efficacité des actes issus de la médiation pour le règlement du litige

La question posée ici est celle de l'efficacité juridique de l'acte issu de la médiation hors instance. A cet égard, il faut souligner d'emblée que la qualification juridique des accords n'est pas différente selon qu'ils sont conclus ou non sous l'égide d'un tiers. Quelle que soit la dénomination retenue pour désigner l'instrumentum (procès-verbal de conciliation, transaction, procès-verbal d'accord), les conventions passées entre les parties à l'issue d'une médiation constituent juridiquement des transactions. La question posée se confond donc avec celle de l'efficacité juridique des transactions. Et cette efficacité peut s'apprécier sous deux aspects: au regard de l'extinction du litige; en termes d'exécution des conventions.

31 Pour la situation britannique, on se reportera au rapport du médiateur Antony Willis au Séminaire sur les Modes Alternatifs de Règlement des litiges. Ce dernier décrit ce qui constitue un véritable marché de la médiation, tant dans le secteur associatif que dans le secteur commercial. Rapport sur l'expérience du Royaume-Uni dans le domaine des modes alternatifs de règlement des litiges », Strasbourg, 29 nov. $1^{\text {er }}$ déc. 1999, doc. CE, SEM:ADR (99)9F. 
Revista Eletrônica de Direito Processual - REDP. Volume 15. Janeiro a Junho de 2015 Periódico Semestral da Pós-Graduação Stricto Sensu em Direito Processual da UERJ. Patrono: José Carlos Barbosa Moreira. www.redp.com.br ISSN 1982-7636 PP 107-149

A- L'extinction du litige par la transaction

Tous les accords transactionnels conclus entre les parties, qu'ils l'aient été ou non sous l'égide d'un médiateur, sont assortis d'une solide efficacité procédurale. Leur effet principal est d'empêcher la saisine d'un tribunal en raison de l'autorité de chose jugée qui s'y attache. L'article 2052 alinéa 1 du code civil pose en ce sens que «les transactions ont, entre les parties, l'autorité de la chose jugée en dernier ressort ». En termes procéduraux, la transaction fait disparaître le droit d'action des parties, qui peuvent dès lors voir opposer une fin de non recevoir à toute demande formée en termes identiques sur les droits initiaux ${ }^{32}$. La transaction s'oppose efficacement à la reprise d'une instance dans les limites de la chose jugée, c'est-à-dire dans le cadre délimité par l'identité de cause, d'objet et de parties, sans que les parties puissent exciper d'une erreur de droit ou de la lésion (article 2052 al.2). Dès lors, la reprise de la contestation initiale ne pourra se faire qu'au prix d'une demande de nullité de la transaction ou en considération du fait que le litige en cause n'était pas compris dans les termes de la transaction.

Si les litiges «primaires" sont ainsi efficacement écartés du tribunal, en revanche, les litiges secondaires peuvent naître de la contestation des accords ou de leur interprétation, litiges qui peuvent être à leur tour portés devant les tribunaux. Les questions juridiques soulevées par la validité et la portée des accords sont du reste parmi les plus complexes qui soient, et la jurisprudence sur ces questions bien difficile à synthétiser. Cette jurisprudence montre en tout état de cause l'importance de la rédaction de l'accord pour définir l'étendue et la portée des obligations. Contrairement à une idée répandue, les transactions sont des contrats complexes qui supposent que soient précisés l'objet du litige, les droits de chacun et les points transigés. Aussi seraitil prudent d'exiger normalement de tout médiateur qu'il incite les parties à donner une forme écrite à leur accord, même si lui-même ne participe pas à la rédaction de l'acte. De plus, l'exigence d'un écrit peut permettre d'envisager l'octroi de délais aux parties, qu'il s'agisse d'un délai de réflexion, intervenant avant l'accord définitif, ou d'un délai de rétractation, ouvert après la conclusion de l'acte.

32 L'article 122 du nouveau code de procédure civile définit la fin de non-recevoir comme « tout moyen qui tend à faire déclarer l'adversaire irrecevable en sa demande, sans examen au fond, pour défaut de droit d'agir, tel le défaut de qualité, le défaut d'intérêt, la prescription, le délai prefix, la chose jugée ». 
Revista Eletrônica de Direito Processual - REDP. Volume 15. Janeiro a Junho de 2015

Periódico Semestral da Pós-Graduação Stricto Sensu em Direito Processual da UERJ.

Patrono: José Carlos Barbosa Moreira. www.redp.com.br ISSN 1982-7636 PP 107-149

B-La force exécutoire de l'accord issu de la médiation

La transaction étant un simple contrat n'est pas en elle-même susceptible d'exécution forcée ${ }^{33}$. En cas de refus ou de difficulté d'exécution, c'est toujours vers le juge qu'il faudra revenir. Ce retour vers un juge qu'on cherchait précisément à éviter peut sembler paradoxal. Mais ce n'est là qu'un rappel des limites de la volonté privée en matière de règlement des litiges. Hors les cas de litiges portant sur la validité du contrat lui-même, le recours au juge visera à l'obtention de l'apposition de la formule exécutoire.

- En France, les dispositions juridiques se multiplient qui tendent à permettre de délivrer un exécutoire pour des transactions conclues hors instance, avec ou sans médiateur. Ainsi, les accords conclus par les commissions de conciliation compétentes en matière d'invention de salariés peuvent être rendus exécutoires par décision du président du tribunal de grande instance (art. L. 615-21 du code de la propriété intellectuelle); de même, devant un conciliateur «si les parties en expriment la volonté dans l'acte constatant leur accord, elles peuvent demander au juge d'instance de donner force exécutoire à l'acte exprimant cet accord», (article 9 du décret du 20 mars 1978); enfin, le décret 98-1231 du 28 décembre 1998 a étendu cette faculté à toutes les transactions, même conclues hors de l'intervention d'un tiers : le juge peut désormais être saisi aux seules fins «de conférer force exécutoire» à une transaction conclue par les parties en-dehors de toute instance (art. 1441-4 du nouveau code de procédure civile).

Le problème est bien évidemment de savoir si ces accords doivent faire l'objet d'un contrôle spécifique de la part du juge. La réponse doit certainement être positive, notamment dans la perspective communautaire d'une libre circulation des titres exécutoires sur le territoire de l'Union européenne 34.

La plus grande attention devrait donc être apportée aux conditions dans lesquelles un tribunal peut être amené à donner force exécutoire à une transaction conclue hors de toute instance. En tout état de cause, ainsi qu'il a précédemment été souligné, la seule intervention d'un médiateur ne saurait constituer une présomption de régularité juridique de l'acte.

33 Sur la comparaison des effets des transactions avec les autres titres tels le jugement et les actes notariés, voir le rapport de synthèse de Jacques Normand au XXIIIème colloque des IEJ sur «L'exécution », Lyon III, 19-20 novembre 1999.

34 Proposition de règlement du Conseil (Com (1999) 384/4), visant à reconnaître de plein droit sur tout le territoire de l'Union le caractère exécutoire des titres, et modifiant la Convention de Bruxelles du 27 septembre 1968. 
Revista Eletrônica de Direito Processual - REDP. Volume 15. Janeiro a Junho de 2015 Periódico Semestral da Pós-Graduação Stricto Sensu em Direito Processual da UERJ. Patrono: José Carlos Barbosa Moreira. www.redp.com.br ISSN 1982-7636 PP 107-149

Deuxième partie

Des médiations à l'ombre du tribunal

Une fois le procès engagé, la médiation ne perd pas de son influence. Il s'agit cette fois non plus d'éviter la saisine d'un tribunal, mais de mettre fin à l'instance sans jugement, et au-delà, de prévenir les litiges ultérieurs. Comme précédemment, cette situation sera envisagée sous les trois points de vue de son l'intérêt économique, de l'adéquation juridique des litiges, et de la validité des accords.

Chapitre 1- Des litiges économiquement adaptés à une médiation en cours d'instance

La médiation est supposée permettre d'éviter les coûts d'une procédure menée jusqu'à son terme. On doit se demander quel avantage financier elle présente par rapport à la décision, et par rapport aux arrangements conclus directement entre les parties.

Section 1 - Avantages comparés de la médiation et de la décision

Lorsqu'une procédure est engagée, la médiation sera économiquement appropriée dans la mesure où elle permet d'économiser les frais d'une procédure poursuivie à son terme, et à condition qu'elle ne génère pas elle-même des coûts propres supérieurs à ceux de la procédure. Or le poids de ces dépenses est fonction de la qualité du tiers intervenant : il sera nul si le juge est lui -même le médiateur; il sera positif si c'est un tiers qui est désigné.

\section{A-Des juges-médiateurs}

Le juge n'est pas un simple pourvoyeur de décisions. Il constitue aussi le médiateur naturel des parties. Certes, les professionnels de la médiation, comme les tenants d'un pluralisme juridique à fondement communautaire, ne manquent pas de critiquer les fonctions conciliatoires assumées par les juges (partialité, absence de pratique de la négociation). Ce courant se déclare hostile de longue date à l'intervention d'un magistrat conciliateur, considérée comme la manifestation d'une «perspective hégémonique du juge»35. Mais les raisons avancées ne sont pas étayées par des comparaisons empiriques, et les motifs des critiques semblent tenir davantage à la

35 V. notamment sur ce point Bonafé-Schmitt, J-P., [1992], « La médiation, une justice douce », Syros alternative . 
Revista Eletrônica de Direito Processual - REDP. Volume 15. Janeiro a Junho de 2015 Periódico Semestral da Pós-Graduação Stricto Sensu em Direito Processual da UERJ. Patrono: José Carlos Barbosa Moreira. www.redp.com.br ISSN 1982-7636 PP 107-149 crainte de voir les juges concurrencer les médiateurs professionnels qu'à des circonstances objectives 36 . Il faut examiner de plus près dans quelles conditions le juge est amené à intervenir comme conciliateur. Si on laisse de côté la mission générale de conciliation qui peut lui être reconnue, et qui ne s'inscrit pas dans une procédure particulière (c'est le cas en France avec l'article 21 du Nouveau code de procédure civile), on observe que les fonctions de conciliateurs sont assurées dans un cadre procédural précis, placé selon les cas avant la saisine du tribunal ou avant le jugement.

-1- La conciliation comme filtre d'entrée au tribunal constitue la forme la plus ancienne de l'intervention du juge conciliateur.

En France, l'article 48 de l'ancien décret de 1806 posait qu'aucune «demande principale introductive d'instance entre parties capables de transiger, et sur des objets qui peuvent être la matière d'une transaction, ne sera reçue dans les tribunaux de première instance, que le défendeur n'ait été préalablement appelé en conciliation devant les juges de paix ou que les parties n'y aient volontairement comparu». Suivait une longue liste de matières «dispensées du préliminaire de conciliation», ce qui attestait du caractère contraignant de ce préalable. Ce rôle de filtre du juge de paix sera maintenu avec ses traits essentiels jusqu'au décret du 22 décembre 1958 .

Ce type d'intervention conciliatoire a aujourd'hui perdu de son intérêt, et peut même être considéré comme une cause inutile d'allongement de la durée si elle constitue une simple formalité préalable, non intégrée à l'instance.

2- Dans sa version moderne, la conciliation menée par le juge peut constituer une phase obligatoire de la procédure, intégrée à l'instance

- Ces phases de conciliation sont nombreuses en France, et trouvent leur terrain d'élection dans les procédures orales: on les trouve devant certains tribunaux (conciliation des conseils de prud'hommes, conciliation des tribunaux paritaires des baux ruraux, tribunaux des affaires de sécurité sociale), ou dans certaines affaires (divorce, saisies arrêts des rémunérations du travail).

36 En Norvège, où un projet pilote a introduit une médiation devant le tribunal en matière civile, les critiques habituellement formées contre l'intervention des juges dans ce domaine semblent être le fait d'un petit nombre de juristes. Engebretsen G., Nestor Nilsen I., "Court mediation in Norway", [1999], Rapport au Séminaire sur la médiation, doc. SEM/ADR (99). 
Revista Eletrônica de Direito Processual - REDP. Volume 15. Janeiro a Junho de 2015 Periódico Semestral da Pós-Graduação Stricto Sensu em Direito Processual da UERJ. Patrono: José Carlos Barbosa Moreira. www.redp.com.br ISSN 1982-7636 PP 107-149

- En Finlande, la réforme de procédure civile entrée en vigueur en décembre 1993 a introduit trois phases dans le procès. La première phase est écrite; la seconde phase est orale, et a lieu devant le juge qui tente d'obtenir un arrangement entre les parties. Ce n'est qu'en cas d'échec de l'arrangement que le cas est transmis à une formation composée de trois juges 37 .

-Dans tous ces cas, la conciliation vise à mettre face à face les parties, mais les juges peuvent également être dotés de pouvoirs juridictionnels spécifiques supplémentaires, comme ordonner la communication de pièces, tirer des conclusions de droit des déclarations des parties, prendre des mesures juridictionnelles urgentes. L'utilité d'une phase de médiation judiciaire, dont il ne faut pas ignorer qu'elle génère un coût en termes de durée de procédure 38 , dépendra en définitive de l'étendue des pouvoirs coercitifs reconnus au juge, et sera d'autant plus grande que ces derniers seront plus étendus.

\section{$B$ - Des médiateurs nommés par le juge}

Le législateur peut être tenté de décharger le juge de ses missions de conciliation, pour des motifs qui relèvent plus du souci de gérer efficacement les moyens des tribunaux que d'améliorer la qualité de la médiation. La conciliation judiciaire est en effet consommatrice de temps de procédure, et il peut apparaître économiquement plus rentable d'inciter le juge à externaliser le temps de la négociation. Mais tout dépend bien sûr de la dépense induite par le recours au médiateur.

1- Cette dépense peut varier selon la procédure. C'est le cas en France, où le médiateur est ou non rémunéré selon le caractère obligatoire ou facultatif de la médiation.

- La loi du 9 février 1995 et des décrets du 22 juillet 1996 et du 28 décembre 1998, autorisent le juge d'instance à déléguer aux conciliateurs de justice la direction des conciliations que la loi met à sa charge 39 . L'accord des parties est requis, et l'intervention du conciliateur est sans frais pour elles.

37 Pour une présentation des conséquences de cette réforme, v. Kaijus Ervasti, [1998],"An assessment of the Reform of Civil Procedure in Finland", Publication of National Research Institute of Legal Policy, $\mathrm{n}^{\circ}$ 154, Helsinki.

38 Devant les Conseils de prud'hommes en France, où existe un préliminaire de conciliation obligatoire, la durée moyenne de procédure est le double de celle du tribunal d'instance $(9,7$ mois contre 5,1 en 1998).

39 Pour une durée d'un mois lorsque le juge est saisi en tentative préalable de conciliation par les parties. (article. 830 et suivants du nouveau code de procédure civile) ; sans formalité et sans durée pour 
Revista Eletrônica de Direito Processual - REDP. Volume 15. Janeiro a Junho de 2015 Periódico Semestral da Pós-Graduação Stricto Sensu em Direito Processual da UERJ. Patrono: José Carlos Barbosa Moreira. www.redp.com.br ISSN 1982-7636 PP 107-149

-Lorsque la conciliation est facultative, un médiateur peut être désigné à tout moment par le juge avec l'accord des parties, «afin d'entendre les parties et de confronter leurs points de vue pour leur permettre de trouver une solution $\gg 40$ Mais cette fois, l'intervention du médiateur comporte un coût pour les parties, tant en termes financier (l'article 131-3 du nouveau code de procédure civile prévoit que le médiateur est rémunéré par les parties, sauf si l'aide juridictionnelle est accordée), qu'en durée de procédure (la mission peut durer trois mois renouvelables une fois). La rémunération est fixée librement par le juge, qui ne prend pas sur ce point l'avis des parties. Face à ce dispositif, quelles conditions doivent être remplies par la médiation pour présenter un intérêt économique?

2- En ce qui concerne les médiations réalisées sans frais par un conciliateur, on peut certes estimer que ce recours au tiers conciliateur économise du «temps magistrat», et donc de la dépense publique. Mais le conciliateur induit lui-même une dépense publique, et la confrontation entre ces deux chefs de dépense ne conduirait pas nécessairement à un bilan positif. Pour les parties, l'intérêt est encore moins évident. En effet, la désignation du conciliateur ne fait qu'ajouter un intermédiaire supplémentaire dont l'intervention vient alourdir la procédure, avec un risque d'allongement de la durée pour les parties. Autrement dit, le temps épargné par le magistrat risque d'être payé en retard de procédure pour les parties, avec un résultat qui n'a aucune raison d'être meilleur que celui qu'obtiendrait le juge.

La médiation judiciaire confiée à un tiers, même sans frais, n'est pas toujours adaptée pour les affaires dont le règlement intervient dans des délais brefs. Or en France, les durées moyenne de procédure devant les juridictions civiles étaient en 1998 de cinq mois pour les tribunaux d'instance et de neuf mois pour les tribunaux de grande instance 41 . C'est dire qu'il apparaît peu rationnel d'ordonner une médiation lorsque le délai de rendu de la décision est aussi court.

3- Quant à la médiation rémunérée, elle est en tout état de cause inadaptée aux litiges de faible valeur, à moins de ne verser au médiateur qu'une rétribution minime.

les procédures simplifiées (art. 847-3 du nouveau code de procédure civile pour les saisines sur requête conjointe, art. 847-3 pour les saisines par simple déclaration au greffe).

40 Titre VI bis du Livre I du nouveau code de procédure civile.

41 Annuaire statistique de la justice, éd. 2000, La Documentation Française, p. 65 
Revista Eletrônica de Direito Processual - REDP. Volume 15. Janeiro a Junho de 2015

Periódico Semestral da Pós-Graduação Stricto Sensu em Direito Processual da UERJ.

Patrono: José Carlos Barbosa Moreira. www.redp.com.br ISSN 1982-7636 PP 107-149

Cette condition de rationalité économique relève du simple bon sens, et on pourrait s'attendre à ce qu'un juge lui-même rationnel ne propose pas une médiation payante dans les litiges de faible importance.

Cependant, il faut prendre garde à ce que certaines circonstances ne conduisent le juge à adopter une attitude non rationnelle à cet égard.

- Ainsi, un juge peut être conduit à proposer une médiation payante si le coût de la médiation n'est pas supporté par les parties, mais par un système d'aide juridictionnelle, ce qui est le cas en France. Sachant qu'en matière civile, l'aide juridictionnelle est accordée surtout dans le secteur de la famille et du divorce, le risque est grand de voir le juge proposer la médiation dans ce secteur, conduisant à un accroissement de l'offre de services de médiation. Autrement dit, le juge peut être amené à proposer une médiation non là où elle est le plus utile, mais là où se trouve une demande solvable.

4- De manière générale, la médiation confiée à des tiers, rémunérés ou non, ne devrait jamais être considérée comme un substitut économiquement viable à une organisation juridictionnelle défectueuse. Ainsi la médiation ne peut jamais être un bon substitut à la décision si les durées moyennes de procédure dans un pays donné sont élevées. Lorsque les durées de procédure sont excessives, on doit s'attacher à améliorer l'efficacité d'ensemble du système juridictionnel, plutôt que de promouvoir des procédés alternatifs qui tendent à pérenniser ces défaillances.

Dans tous les pays, la réflexion menée en termes de «court management» est mieux adaptée à la gestion des instances que toute forme de tentative « d'externalisation » des contentieux par renvoi à des tiers 42 .

Section 2 - Avantages comparés de la médiation et des règlements transactionnels en cours d'instance

En dehors de toute médiation, les procédures se terminent par un règlement non juridictionnel dans des proportions qui varient selon les systèmes juridiques et les types de litiges, mais où le coût de la procédure joue un rôle important.

42 En France, on peut se référer au rapport Francis Casorla [1997], « Réflexions sur l'amélioration de l'accès à la justice par la mise en place d'un guichet unique de greffe et la simplification des juridictions de première instance ». Pour le Royaume -Uni, v. les actes de la conférence qui s'est tenue à Dublin le 16 nov.1996 sur le "Case Management", autour de la présentation du rapport d'un groupe de travail sur les tribunaux mis en place par le Ministre de la justice d'Irlande. (Government Publications, Dublin, 1997). 
Revista Eletrônica de Direito Processual - REDP. Volume 15. Janeiro a Junho de 2015 Periódico Semestral da Pós-Graduação Stricto Sensu em Direito Processual da UERJ. Patrono: José Carlos Barbosa Moreira. www.redp.com.br ISSN 1982-7636 PP 107-149

A-Cô̂t de la médiation et coût de la transaction

L'étude des arrangements intervenus en cours de procédure constitue une partie importante des recherches théoriques en économie de la justice. Dans les modèles construits par les économistes, la variable du coût du procès est centrale pour rechercher quels motifs déterminent les parties à poursuivre une instance jusqu'à son terme ${ }^{43}$. En effet, le coût de la procédure constitue un motif puissant d'abandon prématuré de l'instance, et il faut rechercher les explications qui peuvent conduire les parties à adopter le comportement non rationnel de poursuite de l'instance. La présence de l'avocat étant toujours présupposée, les économistes s'attachent à étudier les processus transactionnels menés par l'intermédiaire des conseils. La réputation des avocats en matière de préférence transactionnelle figure du reste parmi les variables contribuant à accroître les chances d'un règlement transactionnel, au même titre que le type d'honoraires 44 .

En revanche, ces modèles ne trouvent plus à s'appliquer lorsqu'il faut intégrer les frais d'une médiation: l'intérêt économique de la négociation n'est plus assuré s'il faut ajouter aux honoraires des conseils (qui restent dus en tout état de cause), ceux d'un médiateur. L'intervention d'un tiers dans ce jeu de négociations ne ferait qu'accroître la complexité de la situation, en soulevant le problème de la participation des avocats à l'entreprise de médiation.

Sans entrer dans le détail de ces raisonnements modélisés, il faut retenir que la médiation doit toujours être abordée dans son environnement judiciaire, c'est-à-dire dans un espace d'actions où se trouvent déjà présents les parties, leurs conseils, les experts et les juges. Ainsi, dans les procédures avec représentation obligatoire, on doit considérer que ce sont les conseils qui pourraient assumer les fonctions de négociation. A défaut de conseils, la médiation rémunérée ne doit intervenir que si le juge n'est pas lui-même en mesure de mener une conciliation.

\section{B-La médiation et la probabilité d'accord entre les parties}

Dans des juridictions comme l'Angleterre et le pays de Galles où les coûts de procédure sont très élevés, l'incitation à l'abandon des instances ou le règlement des

43 Pour une présentation d'ensemble des différents modèles économiques établis sur la décision de poursuite du procès, v. Doriat-Duban M. [2000], "L'analyse économique du règlement des conflits: application au droit civil français", Thèse de doctorat en sciences économiques, Université de Nancy II.

44 Hay B.L., Spier K.E., [1998], "Settlement of litigation", in Newman, P., The New Palgrave Dictionary of Economics of the Law, vol. 3, London, Macmillan. 
Revista Eletrônica de Direito Processual - REDP. Volume 15. Janeiro a Junho de 2015 Periódico Semestral da Pós-Graduação Stricto Sensu em Direito Processual da UERJ. Patrono: José Carlos Barbosa Moreira. www.redp.com.br ISSN 1982-7636 PP 107-149 litiges avant jugement est forte. Les taux d'abandon ou de règlement du litige avant jugement dépassent $90 \% 45$. En France, les fins d'affaires sans jugement varient en fonction de la nature des litiges 46 . Ainsi, $45 \%$ des litiges consécutifs à la rupture du contrat de travail ne vont pas jusqu'au jugement, ainsi que 32\% des affaires de responsabilité médicale 47 . A l'inverse, les demandes en paiement contractuelles ne sont abandonnées que dans $12 \%$ des cas. Devant des taux d'arrangement élevés, quel intérêt supplémentaire peut présenter une médiation?

- Dans les litiges où les taux d'arrangement directs entre les parties sont les plus forts, il peut apparaître peu productif de faire intervenir un médiateur rémunéré. Procéder à l'inverse reviendrait à «voler au secours de la victoire », et à induire des surcoûts inutiles pour la collectivité comme pour les parties 48 .

Par ailleurs, on notera que nombre de ces litiges à fort taux de transaction requièrent l'intervention d'experts. On peut estimer que la présence d'experts dans un contentieux rend inutile le recours à un tiers, ce qui relance le débat de la possibilité pour l'expert d'établir lui-même un procès-verbal de conciliation.

En cours d'instance, la médiation payante devra être proposée seulement dans les domaines à faible niveau de négociation, où les intérêts financiers en jeu sont suffisamment élevés, et en l'absence de conseils ou d'experts aptes à mener une négociation.

Chapitre 2 Des litiges juridiquement adaptés à une orientation vers des médiations en cours d'instance

En matière civile, l'instance appartient aux parties. De même qu'elles étaient libres d'en introduire une, elles sont libres d'y renoncer, l'abandon de la procédure étant

45 C'est ce taux qu'évoquait un des participants au séminaire sur le «Case management», le Pr. Michael Zander.

46 Le tableau ci-dessous met en évidence ces disparités pour la France.

Résultat selon les juridictions(1998)FondTotal affaires terminées Dont sans décision juridictionnelle\% des décisions jon juridictionnellesConseils de prud'hommes $1659477495645,1 \%$ Tribunaux de commerce 1680383301919,6\%Tribunaux

d'instance4526469823921,7\%Tribunaux de grande instance63534015107323,7\% Source : Répertoire général civil, Ministère de la Justice.

47 Données fournies pour 1997 par le Répertoire général civil.

48 Cette inquiétude est partagée par certains observateurs du système de médiation, comme Lord Mustill, ([1996] (Judicial Processes and Alternative Dispute Resolution, Israël Law Review,,vol. 30, n¹2.). Ce dernier évoquait le cas des affaires où un accord serait intervenu de toutes façons et où les parties peuvent se voir inciter à accepter un règlement inéquitable. 
Revista Eletrônica de Direito Processual - REDP. Volume 15. Janeiro a Junho de 2015 Periódico Semestral da Pós-Graduação Stricto Sensu em Direito Processual da UERJ. Patrono: José Carlos Barbosa Moreira. www.redp.com.br ISSN 1982-7636 PP 107-149 constaté par un des actes que prévoient les codes de procédure civile (désistement, transaction en cours d'instance, péremption, caducité). Mais peut-on considérer qu'un Etat peut conduire une politique systématique d'incitation à l'abandon des procédures? La réponse n'est pas évidente. Comme l'a montré une enquête menée auprès des juges finlandais en 1997, «l'augmentation du nombre de cas qui ont été réglés par médiation peut être jugée positive, bien que du point de vue de la légalité, un doute puisse être émis sur le maintien des garanties du procès équitable dans des procédures moins formelles ${ }^{49}$. Si un tribunal a été saisi, c'est que le motif d'agir du demandeur a été suffisamment déterminant pour lui permettre de surmonter son aversion au risque. Et si la procédure s'est poursuivie, c'est que la décision a constitué, pour une raison ou pour une autre, un objectif souhaitable pour au moins une des deux parties.

La médiation judiciaire ne pourra donc être encouragée que là où les droits sont disponibles. Et là où les droits sont disponibles, le type de médiation doit entrer également en considération.

Section 1 - Des droits juridiquement adaptés à la négociation

Tous les droits ne sont pas également disponibles, et seront donc inégalement négociables. Certains sont toujours indisponibles (A). D'autres ne le sont que partiellement (B).

\section{A- Des droits non négociables}

Les droits indisponibles ne peuvent faire l'objet d'une transaction entre les parties, et ne sont donc pas adaptés à la médiation. Reste à déterminer quels droits sont indisponibles. Un indicateur peut être retenu, celui du caractère obligatoire de la décision d'un juge lorsqu'il s'agit de disposer de certains droits. Lorsqu'un acte juridique concernant un droit ne peut être valablement accompli que sous contrôle d'un juge, la médiation concernant ce droit apparaît inadaptée.

C'est le cas dans la procédure civile française pour tous les droits qui entrent dans la matière gracieuse, définie par l'article 25 du nouveau code comme celle où le juge « statue lorsqu'en l'absence de litige il est saisi d'une demande dont la loi exige en raison de la nature de l'affaire ou la qualité du requérant, qu'elle soit soumise à son contrôle». Les affaires entrant dans la matière gracieuse sont nombreuses, et constituent une part importante de l'activité des juges spécialisés. On y trouve des actes relatifs à l'état des

49 Kaijus E., [1998], op. cit. 
Revista Eletrônica de Direito Processual - REDP. Volume 15. Janeiro a Junho de 2015 Periódico Semestral da Pós-Graduação Stricto Sensu em Direito Processual da UERJ. Patrono: José Carlos Barbosa Moreira. www.redp.com.br ISSN 1982-7636 PP 107-149

personnes (divorces sur demandes conjointes, adoption), aux personnes incapables (tutelle des mineurs et des majeurs), certains aspects patrimoniaux du mariage (changements de régimes matrimoniaux), l'autorité parentale (assistance éducative, déclaration conjointe d'autorité parentale à l'égard des enfants naturels etc...).

\section{$B$-Des droits négociables sous contrôle}

-En matière contentieuse, certains droits ne peuvent faire l'objet de conventions que dans des limites étroites. Il en est ainsi de l'obligation d'entretien des parents à l'égard de leurs enfants. Les conventions concernant ces droits doivent préserver l'intérêt des enfants, et notamment, ne pas contenir des renonciations qui pourraient léser leurs intérêts.

Mais qui dit restriction du pouvoir d'agir dit contrôle par une autorité supérieure. Cette autorité peut être une administration. Mais la plupart du temps, c'est le juge qui sera désigné. Quoiqu'il en soit, le médiateur n'a plus sa place dès lors que le litige ne met pas seulement en jeu les intérêts des parties présentes, mais également ceux des tiers ou de la collectivité.

Ainsi, l'opportunité de l'intervention d'un médiateur dans un litige entre les parents relativement à la fixation d'une pension ne peut se situer qu'à la marge de l'intervention judiciaire. Le médiateur peut certes amener les parties à s'entendre, mais il reviendra en tout état de cause au juge de vérifier que ces accords ne portent pas atteinte aux droits des enfants au bénéfice d'une contribution, ce qui réduit l'extension et donc l'intérêt d'une mission de médiation.

-Dans d'autres domaines contentieux, le législateur édicte des règles d'ordre public qui visent à protéger les parties considérées comme les plus faibles: le salarié dans ses relations avec l'employeur, le preneur d'un bail d'habitation à l'égard du bailleur, l'emprunteur vis-à-vis du prêteur, la victime d'un accident de la circulation à l'égard de l'assureur. Ces droits qui relèvent d'un ordre public dit « de protection » ne deviennent disponibles qu'après être entrés dans le patrimoine de la partie protégée, c'est-à-dire après la survenance d'un litige. L'incitation à une médiation en cours de procédure, sans contrôle sur le fond de ces droits, risque de réintroduire un déséquilibre que les politiques législatives visaient précisément à corriger. Aussi dans les domaines où les droits font l'objet d'une protection spéciale, la médiation judiciaire ne devrait pas être encouragée. La préférence doit être donnée à ces instances de conciliation spécialisées intervenant en amont de la saisine du tribunal, dont nous avons donné des 
Revista Eletrônica de Direito Processual - REDP. Volume 15. Janeiro a Junho de 2015 Periódico Semestral da Pós-Graduação Stricto Sensu em Direito Processual da UERJ. Patrono: José Carlos Barbosa Moreira. www.redp.com.br ISSN 1982-7636 PP 107-149 exemples en première partie. Si un litige naît à la suite de l'intervention de ces commissions, ce sera au juge de trancher, sans pouvoir désigner un nouveau médiateur, qui serait en tout état de cause moins bien informé que la commission.

Section 2 - Les formes de médiation judiciaires adaptées aux litiges négociables

Dans les litiges dont l'objet est totalement disponible pour les parties, l'alternative se situe entre confier des pouvoirs de médiation au juge et les déléguer à un tiers, rémunéré ou non. Si on laisse de côté toute considération financière pour ne s'interroger que du point de vue de l'intérêt juridique, le choix doit se faire par comparaison entre les missions de conciliation dévolues respectivement au juge et au tiers. A cet égard, on peut affirmer que les devoirs des uns et des autres ne sont pas identiques.

- Le tiers médiateur n'a pas a priori d'autre mission que celle de rapprocher le point de vue des parties, et les considérations d'application de la loi ne peuvent pas être déterminantes dans son action, sauf à contraindre le médiateur à inciter les parties à une stricte application de la loi. On ne peut donc envisager de lui confier le soin de rapprocher des points de vue dans des litiges où sont en cause des droits non disponibles ou dont la complexité suppose qu'une information exacte soit donnée aux parties.

- Le juge au contraire est doté de la double fonction de dire le droit et de concilier les parties. Lorsqu'il statue dans le cadre de ses fonctions juridictionnelles, et sauf dispense conventionnelle ou légale, il fait application des règles de droit 50 . Doit-on considérer que lorsqu'il intervient comme juge médiateur, il est désormais dispensé de faire référence aux règles de droit, qu'il s'agisse des règles de procédure ou des règles de fond? Une telle conclusion serait paradoxale. On doit considérer au contraire que lorsque le juge intervient comme conciliateur, il est mieux placé que quiconque pour mener la négociation de manière indépendante et impartiale, et contribuer à la conclusion d'un accord équitable. Il importe d'inciter le juge conciliateur à agir dans ce sens, ce qui implique que soient instaurées des règles procédurales minimales. De plus, les actions prévues par la loi en matière de responsabilité de l'Etat pour fonctionnement

$50 \quad$ En France, les parties peuvent donner au juge par convention le pouvoir de statuer en amiable composition (art. 12 al. 4 du nouveau code de procédure civile). En Italie, le juge de paix qui statue dans des causes dont la valeur est inférieure à deux millions de lires, et à condition que la partie intéressée ne s'y oppose pas, peut décider en équité sans suivre strictement la règle de droit. 
Revista Eletrônica de Direito Processual - REDP. Volume 15. Janeiro a Junho de 2015 Periódico Semestral da Pós-Graduação Stricto Sensu em Direito Processual da UERJ. Patrono: José Carlos Barbosa Moreira. www.redp.com.br ISSN 1982-7636 PP 107-149 défectueux du service public de la justice pourraient s'appliquer à l'activité conciliatoire du juge.

Chapitre 3-Des litiges efficacement réglés par une médiation en cours d'instance

L'efficacité juridique de la médiation ne se mesure pas en nombre d'accords conclus, mais dans son aptitude à produire des actes juridiques eux-mêmes efficaces, c'est-à-dire qui permettent de clôturer l'instance, et dont la validité est garantie.

Section 1 - L'efficacité des actes issue de la médiation sur la clôture du litige

En raison des délais et des coûts induits par une médiation, les litiges qui doivent lui être renvoyés de manière préférentielle sont ceux où l'intervention du médiateur peut mettre fin efficacement à l'instance introduite en déliant le juge de son obligation de juger.

\section{.A-Des accords qui éteignent l'instance}

Un des critères de l'efficacité est de permettre le règlement total et immédiat de la cause du litige entre les parties intéressées. Or tous les litiges ne se prêtent pas également à un règlement définitif et complet des causes du différend. Les litiges complexes, comme les procédures de divorce ou les demandes consécutives à une rupture du contrat de travail, sont susceptibles d'être décomposés en éléments plus petits qui peuvent faire l'objet d'arrangements partiels.

Dans quelle mesure la médiation est-elle adaptée à la recherche d'accords partiels? La procédure civile française en admet le principe pour la conciliation menée par le juge: l'article 130 du nouveau code de procédure civile (applicable devant toutes les juridictions), indique qu'en cas de conciliation menée par le juge, la teneur de l'accord, même partiel, est constatée dans un procès-verbal signé par le juge et les parties ». La médiation confiée à un tiers peut dans le même sens «porter sur tout ou partie du litige» (art. 131-2), ce qui implique que l'accord peut être partiel. Pour des raisons pratiques, cette faculté juridique doit cependant être maniée avec discernement.

Si la médiation menée par le juge peut sans dommage avoir un objet limité, dans la mesure où le juge conserve une maîtrise de la gestion de l'instance, il convient d'être vigilant dans l'usage des missions de médiations partielles confiées à des tiers, qui peuvent différer inutilement le moment du règlement définitif de l'ensemble du litige. De 
Revista Eletrônica de Direito Processual - REDP. Volume 15. Janeiro a Junho de 2015 Periódico Semestral da Pós-Graduação Stricto Sensu em Direito Processual da UERJ. Patrono: José Carlos Barbosa Moreira. www.redp.com.br ISSN 1982-7636 PP 107-149 plus, ces médiations partielles peuvent créer des difficultés d'appréciation par le juge de ce qui «reste à juger». En effet, il est bien difficile d'imaginer de régler un différend sur la résidence du mineur, sans régler en même temps l'ensemble des conséquences juridiques qui s'attachent à cette résidence (pension alimentaire, droits de visite, allocation du logement pour la résidence de la famille).

Il en résulte que la médiation confiée à un tiers doit être préférée là où l'accord à conclure est à même d'épuiser la matière litigieuse.

\section{$B$-Des accords qui préviennent des litiges à venir}

Un thème récurrent des promoteurs de la médiation, notamment en matière familiale, est qu'elle permettrait plus efficacement qu'un jugement d'empêcher la survenance de litiges ultérieurs, en raison de son aptitude à pacifier les relations entre les parties. Cette hypothèse d'efficacité repose sur des prémisses psychologiques qui mériteraient d'être mises à l'épreuve d'une recherche dont la conception reste à faire. Ce qui peut en revanche être juridiquement évalué, c'est l'aptitude d'un litige à être négocié de manière durable, c'est-à-dire au-delà de l'instance en cours. L'effet préventif de l'accord va s'exercer sur différents plans.

1- Il faut tout d'abord rappeler que l'accord conclu sous l'égide d'un médiateur et homologué ou constaté par le juge, bénéficie de la force exécutoire. En revanche, si l'accord n'est pas soumis au juge, l'instance se terminera par une simple décision de dessaisissement qui constatera un désistement.

2- Si l'accord conclu entre les parties est de ceux qui épuisent la matière litigieuse, les voies de recours ne seront pas ouvertes contre la décision qui se borne à en prendre acte. Il en résulte un effet préventif de la médiation sur l'appel. Mais cet effet s'attache à tout règlement non juridictionnel, qu'il ait été ou non conclu sous l'égide d'un tiers.

3- L'accord peut également exercer un effet préventif sur les litiges ultérieurs, si l'acte conclu intègre des prévisions sur l'évolution probable de la situation qui fait l'objet du contrat. Ainsi de l'aggravation de l'état de santé d'une personne en cas de dommages corporels, des pertes futures en matière de dommages économiques, etc...Faute de prévision spéciale sur tel ou tel élément du litige «la transaction se renferme dans son objet » (art. 2048 du code civil), ce qui signifie que ce qui n'aura pas été prévu pourra faire l'objet d'une contestation ultérieure. Non seulement un écrit doit être rédigé à l'issue de toute médiation, mais la rédaction doit en être menée avec un 
Revista Eletrônica de Direito Processual - REDP. Volume 15. Janeiro a Junho de 2015 Periódico Semestral da Pós-Graduação Stricto Sensu em Direito Processual da UERJ. Patrono: José Carlos Barbosa Moreira. www.redp.com.br ISSN 1982-7636 PP 107-149

soin tout particulier. Il importe de prendre conscience que cet écrit constitue un contrat pour les parties, et que ce contrat est exécutoire.

4- Lorsque le litige dont est saisi un tribunal est de ceux qui sont susceptibles de connaître de constantes modifications dans le temps, la médiation apparaît comme peu efficace. Il en est ainsi dans le domaine de la révision des pensions alimentaires. La Cour de cassation française rappelle constamment que les conventions relatives à la contribution à l'entretien et l'éducation des enfants pouvaient faire l'objet de révision dès lors que des changements sont intervenus Le fait qu'une pension ait été contractuellement fixée (comme dans le cas d'une convention de divorce), ne change rien à ce droit de demander la révision; dont la cause est une modification de la situation des parties.

Section 2 - Des droits efficacement préservés par le recours à la médiation

Le médiateur qui intervient dans un litige doit être en mesure d'obtenir un accord efficace au regard de la réalisation des droits des parties.

A-Adéquation de la médiation à l'objectif visé

- L'efficacité du choix de la médiation peut d'abord être évaluée en termes d'adéquation à l'objectif visé. La Cour Européenne des droits de l'homme veille à cet égard à l'efficacité du choix de la médiation par rapport à des mesures coercitives. Dans son l'arrêt Hokkanen c.Finlande, rendu le 23 septembre 1994, la Cour Européenne des droits de l'Homme a condamné l'Etat finlandais sur le fondement de l'article 8 de la convention (droit au respect de la vie familiale), pour n'avoir pas pris les mesures propres à garantir le respect des droits parentaux. Un père qui avait provisoirement remis sa fille à ses beaux- parents après le décès de sa femme n'avait pu obtenir sa restitution, malgré une série de décisions de justice prises en sa faveur. A ces décisions avaient été opposées plusieurs mesures de médiation qui toutes avaient échoué, aggravant la situation initiale 51 . Le respect des droits parentaux exigeait aux yeux de la

51 CEDH, Hokkanen c. Finlande, 23 septembre 1994, n 50/1993/445/524. En l'espèce, la loi finlandaise prévoyait une médiation pour l'exécution forcée des mesures relatives à la garde et au droit de visite. Mais ce même texte précisait que «la conciliation ne doit pas être ordonnée si des tentatives antérieures montrent qu'elle serait vouée à l'échec ou dans le cas d'une décision concernant la garde, s'il est de l'intérêt de l'enfant que cette décision soit, pour des raisons sérieuses, exécutée immédiatement ». 
Revista Eletrônica de Direito Processual - REDP. Volume 15. Janeiro a Junho de 2015 Periódico Semestral da Pós-Graduação Stricto Sensu em Direito Processual da UERJ. Patrono: José Carlos Barbosa Moreira. www.redp.com.br ISSN 1982-7636 PP 107-149

Cour que soient prises des «mesures adéquates » pour faciliter la réunion, la médiation n'étant pas considérée en l'espèce comme une mesure appropriée.

Cet exemple montre que la décision de recourir à une médiation n'est pas toujours anodine, et qu'elle peut conduire à mettre en péril des droits légitimes. Du point de vue juridique, il peut donc être utile de prévoir des mesures de sauvegarde, comme le fait que la médiation ordonnée constitue un acte interruptif de la prescription. De plus, une décision de recourir à une médiation ne doit jamais être imposée en présence d'une situation d'urgence ou d'atteinte illégitime à un droit.

\section{$B$-Validité juridique de l'acte issu de la médiation}

L'efficacité de la médiation s'entend également et surtout de son aptitude à produire des actes juridiques valides.

Ainsi ne sera pas juridiquement efficace un accord conclu sous l'égide d'un médiateur, qu'il soit juge ou tiers agissant sur mandat, et qui lèse une partie. Deux exemples de cette absence d'efficacité juridique de la transaction peuvent être donnés, tirés de la jurisprudence française. Un arrêt de la Chambre Sociale du 28 mars 2000 a précisé les obligations des juges prud'homaux (juges du travail) lorsqu'ils interviennent comme conciliateurs : «la conciliation, préliminaire obligatoire de l'instance prud'homale, est un acte judiciaire qui implique une participation active du bureau de conciliation à la recherche d'un accord des parties préservant les droits de chacune d'entre elles; en conséquence, cet acte ne peut être valable que si le bureau a rempli son office en ayant, notamment, vérifié que les parties étaient informées de leurs droits respectifs »52, en conséquence de quoi «l'accord constaté par le procès-verbal de conciliation est nul ». La troisième Chambre civile a considéré dans le même sens qu'est inefficace un procès-verbal de conciliation signé devant un tribunal et engageant les intérêts des deux époux alors qu'un seul 1'a signé 53 .

Ces deux exemples montrent que le contrôle de validité des transactions ne s'arrête pas à l'étape de la conclusion de l'accord et de la délivrance de la formule exécutoire. La médiation constitue, quoiqu'en disent ses promoteurs, une activité dont les implications sont tout autant juridiques que sociales, en ce qu'elle engage des droits

52 Cour de cassation, Chambre Sociale, 28 mars 2000, Bulletin civil, Bulletin d'information, arrêt $\mathrm{n}^{\circ} 1808$.

53 Cour de cassation, Troisième Chambre Civile, 24 juin 1992, pourvoi $\mathrm{n}^{\circ}$ 91-10.506, Lamy $\mathrm{n}^{\circ}$ 1140. Il s'agissait en l'espèce d'une conciliation effectuée par le tribunal paritaire des baux ruraux. 
Revista Eletrônica de Direito Processual - REDP. Volume 15. Janeiro a Junho de 2015 Periódico Semestral da Pós-Graduação Stricto Sensu em Direito Processual da UERJ. Patrono: José Carlos Barbosa Moreira. www.redp.com.br ISSN 1982-7636 PP 107-149 et implique de procéder à une opération complexe d'appréciation de l'équilibre des prestations.

Cette conclusion rejoint celle que nous avons pu tirer à propos de l'efficacité juridique de la médiation hors instance. Qu'elle ait lieu hors du tribunal ou en cours de procédure, qu'elle soit menée par un juge ou par un tiers, la médiation ne constitue pas cette « justice douce », qui, comme les médecines du même nom, ne saurait jamais nuire à ses utilisateurs. Il en résulte que ses usagers doivent bénéficier des mêmes garanties d'indépendance et d'impartialité que celles qui sont offertes à l'usager d'un tribunal.

Toutes ces observations contredisent les propos de ceux qui prônent la médiation sans droit, et considèrent que « le codage juridique présente des effets aliénants pour les parties $54_{«}$. On ne peut en effet oublier que l'Etat doit avant tout à ses citoyens la garantie de leurs droits. On ne peut non plus oublier que le conflit s'alimente des droits qui en forment la matière.

Sur ce point, la formule de Julien Freund reste plus que jamais d'actualité: le droit est tout autant le motif que la solution des conflits 55 .

Résumé des observations relatives à la médiation civile

I - Les litiges adaptés à une médiation antérieure à la saisine d'un tribunal

1-1 Observations relatives à l'intérêt économique d'une médiation hors instance

1- Une médiation financée par les parties est économiquement adaptée aux litiges pour le règlement desquels n'existent pas des procédures rapides et peu coûteuses.

2-Néanmoins, une médiation financée par la collectivité peut être rendue obligatoire dans les litiges pour le règlement desquels existent des procédures rapides et peu coûteuses, s'il apparaît que les intérêts des défendeurs méritent de faire l'objet d'une protection particulière.

3- Une médiation financée par les parties est économiquement adaptée aux litiges dont la valeur, actuelle ou attendue, est élevée, et parmi ces derniers, à ceux qui sont arbitrables.

54 De Munck, J., [1998], « De la loi à la médiation », in Les révolutions invisibles, Calmann-Levy. 55 Freund, J. [1974], «le droit comme motif et solution des conflits», Archiv für Rechts und Sozialphilosophie, p. 47-62. 
Revista Eletrônica de Direito Processual - REDP. Volume 15. Janeiro a Junho de 2015 Periódico Semestral da Pós-Graduação Stricto Sensu em Direito Processual da UERJ. Patrono: José Carlos Barbosa Moreira. www.redp.com.br ISSN 1982-7636 PP 107-149

4- Une médiation financée par la collectivité doit être encouragée dans les litiges où les demandeurs ne sont pas incités à agir.

5- Dans les litiges où les chances d'un règlement négocié dépendent de la bonne volonté d'une partie économiquement puissante, la mise en place d'une procédure transactionnelle obligatoire entre les parties est préférable à la médiation.

1-2 Observations relatives à l'orientation juridique des litiges vers la médiation hors instance

1- La médiation généraliste pourra être encouragée à l'égard des Pays qui ne disposent pas de moyens pour mettre en place des médiations spécialisées.

2- La médiation spécialisée devrait être encouragée pour traiter des affaires complexes.

3- Les Etats doivent être encouragés à mettre gratuitement à la disposition des usagers des commissions de médiation spécialisées, notamment partout où les parties en présence sont de force inégale, dans des conditions qui garantissent l'équilibre des intérêts et l'application du droit en cause dans les litiges.

4- Si les clauses de médiation prévues dans un contrat sont obligatoires pour les parties, elles ne peuvent, à l'égard des non professionnels, avoir pour effet de faire obstacle à la saisine d'un tribunal.

1-3- Observations relatives à l'efficacité juridique des accords issus d'une médiation

1-Pour préciser l'objet et la portée de l'accord, un écrit devrait normalement être établi à l'issue de toute médiation, et un délai octroyé aux parties, soit sous forme de délai de réflexion placé avant l'accord définitif, soit comme délai de rétractation ouvert après la conclusion de l'acte.

2- Le médiateur doit être en mesure de répondre des conséquences de son intervention, en contractant une assurance de responsabilité s'il est indépendant ou en engageant la responsabilité de l'Etat s'il intervient dans un cadre public.

3- L'octroi de la force exécutoire à un accord issu d'une médiation hors instance doit faire l'objet d'un contrôle du juge, notamment dans la perspective d'une libre circulation des titres exécutoires sur le territoire de l’Union. 
Revista Eletrônica de Direito Processual - REDP. Volume 15. Janeiro a Junho de 2015 Periódico Semestral da Pós-Graduação Stricto Sensu em Direito Processual da UERJ. Patrono: José Carlos Barbosa Moreira. www.redp.com.br ISSN 1982-7636 PP 107-149

II - Les litiges adaptés à une médiation en cours d'instance

2-1 -Observations relatives à l'intérêt économique d'une médiation en cours d'instance

1- Les phases préalables de conciliation menées par le juge doivent être introduites ou maintenues si elles présentent une utilité en termes de mise en état des affaires ainsi que pour l'adoption de mesures urgentes.

2.- La médiation confiée à un tiers dont la rémunération est à la charge des parties ne devrait pas être imposée pour les affaires dont le règlement intervient dans des délais brefs, ou pour les litiges de faible valeur.

3- Dans ce type de litiges, la médiation confiée à un tiers dont la rémunération est à la charge des parties ne devrait pas faire l'objet d'une prise en charge financière au titre de l'aide juridique.

4- De manière générale, la médiation confiée à des tiers, rémunérés ou non, ne doit jamais être considérée comme un substitut économiquement viable à une organisation juridictionnelle défectueuse.

5- Dans les procédures avec représentation obligatoire, il revient aux conseils des parties d'assumer les fonctions de négociation. A défaut de conseils, la médiation rémunérée ne doit intervenir que si le juge n'est pas lui-même en mesure de mener une conciliation.

6- En cours d'instance, la médiation dont la rémunération est à la charge des parties devra être réservée aux litiges qui connaissent les plus faibles niveaux de négociation, à condition toutefois que l'intérêt financier soit suffisant.

2-2 -Observations relatives à l'adéquation juridique des litiges à une médiation en cours d'instance

1- Lorsqu'un acte juridique concernant un droit ne peut être valablement accompli que par un juge, la médiation concernant ce droit apparaît inadaptée.

2- La médiation n'est plus adaptée dès lors que le litige ne met pas seulement en jeu les intérêts des parties présentes, mais également ceux des tiers ou de la collectivité.

3- Dans les domaines où des droits font l'objet d'une protection spéciale, la médiation judiciaire ne devrait pas être encouragée même si les droits sont devenus disponibles. 
Revista Eletrônica de Direito Processual - REDP. Volume 15. Janeiro a Junho de 2015

Periódico Semestral da Pós-Graduação Stricto Sensu em Direito Processual da UERJ. Patrono: José Carlos Barbosa Moreira. www.redp.com.br ISSN 1982-7636 PP 107-149

4- Lorsque la médiation est réalisée par un juge, elle doit être menée de manière indépendante et impartiale, dans un cadre procédural comportant des garanties minimales.

2-3 -Observations relatives à l'efficacité juridique des accords issus d'une médiation en cours d'instance

1- Les missions de médiation partielles confiées à des tiers doivent être évitées dès lors qu'elles risquent de différer excessivement le moment de règlement définitif du litige.

2- L'acte issu de la médiation devrait être normalement écrit, et prévoir l'évolution de la situation litigieuse actuelle.

3- La médiation ne devrait pas se substituer à une décision rendue nécessaire par la situation d'urgence ou d'atteinte illégitime à un droit.

4- Un accord conclu sous l'égide d'un médiateur, qu'il soit juge ou tiers, doit toujours pouvoir faire l'objet d'une action en nullité pour les causes prévues en droit des contrats. 